\title{
Control of coal facies to adsorption-desorption divergence of coals: A case from the Xiqu Drainage Area, Gujiao CBM Block, North China
}

\author{
Long Zhao ${ }^{\mathrm{a}}$, Yong Qin ${ }^{\mathrm{b}, *}$, Chunfang Cai ${ }^{\mathrm{a}}$, Yiwei Xie ${ }^{\mathrm{b}, \mathrm{c}}$, Gang Wang ${ }^{\mathrm{d}}$, Bo Huang ${ }^{\mathrm{b}}$, Chenlu Xu ${ }^{\mathrm{a}}$ \\ ${ }^{a}$ Key Laboratory of Petroleum Resources Research, Institute of Geology and Geophysics, Chinese Academy of Sciences, Beijing 100029 , \\ China \\ ${ }^{\mathrm{b}}$ Key Laboratory of Coalbed Methane Resources and Reservoir Formation Process, Ministry of Education, China University of Mining \\ and Technology, Xuzhou, Jiangsu 221116, China \\ ${ }^{\mathrm{c}}$ Geology Section of Xishan Coal-Electricity Group Co., Ltd., Taiyuan, Shanxi 030053, China \\ ${ }^{\mathrm{d}}$ School of Earth Science and Engineering, Hebei University of Engineering, Handan, Hebei 056038, China
}

\begin{abstract}
Adsorption and desorption of coal to methane are substantially related to coal facies, referring to the production potential of a coalbed methane (CBM) well. Based on analyses of the stratified channel samples from coal seams No. 2, No. 4 and No. 9 in the Xiqu Drainage Area, Gujiao CBM Block, North China, the relationship between coal facies and adsorption-desorption behaviors was first proposed and discussed. Using the coal facies diagrams suggested by previous investigators, four types of coal facies were distinguished in the coal seams, including the upper delta plain wet forest swamp (I), the lower delta plain fen (II), the lower delta plain marsh (III) and the back barrier low moor (IV). The upper delta plain wet forest swamp occurred dominantly, developing at the bottom and top of all the coal seams. Both the ash yield and groundwater index (GWI) tend to increase from coal facies I to IV, whereas the volatile compounds, fixed carbon, organic sulfur, total sulfur, micropore volume and specific surface area, tissue preservation index (TPI) and vegetation index (VI) decreased, which indicates changes in the vegetation types, water supplements and disturbance, acid-base properties and redox conditions in the paleomires. Paleomire evolution accompanied the rise and drop of the sea level, with a transgression or regression process that could be represented by the coal facies sequence. Coal seams No. 2, No. 4 and No. 9 were discriminated into two, three and two evolving stages, respectively, and these stages were characterized by complete cycles, except stage 4-I from the lower delta plain marsh. Mudstone partings in the coal seams deposited during the highest sea level. Collectively, paleomire environments, especially the variation of vegetation types and mire water condition, result in adsorption and desorption divergences among different coal facies. Arborescent plants decrease from coal facies I to IV as the hydrodynamic intensity increases, leading to corresponding changes in botanical tissue preservation, medium oxidizability, mineral content and, ultimately, adsorption and desorption behaviors. In view of the gas content and desorption capacity of the coal layers in the same coal seam, the layers from the upper delta plain wet forest swamp may have the best potential for CBM drainage, followed by those from the lower delta plain fen and marsh, while those from the back barrier low moor may have the worst potential.
\end{abstract}

Keywords: coal facies; paleomire; adsorption; desorption; Gujiao Block

\section{Introduction}

As an emerging clean energy with a huge reserve, coalbed methane (CBM) is the unconventional gas resource that is most likely to implement industrial exploitation (Flores, 1998). CBM productivity and recovery efficiency are directly affected by coal's adsorption-desorption behaviors, which are mainly influenced by the substance composition and structure of coal determined by the depositional setting and paleomire type. However, the particular

${ }^{*}$ Corresponding author. Tel/Fax: +8651683590070

E-mail address: yongqin@cumt.edu.cn (Qin Yong). 
relevance between the facies and adsorption-desorption behaviors of coal has seldom been researched before now.

Coal facies, as the original genetic types of coal formed in given paleomires, have drawn extensive attention since the 1950s (Han et al., 1996). Research on the types of peat accumulation, plant community, depositional conditions, redox potential and geochemistry may provide information about coal facies and its evolution (Dai et al., 2007). Coal petrography, palynology, paleobotany and organic geochemistry are the main methods used to define the coal facies or the depositional environment of peat (Teichmüller, 1989; Nowak and Górecka-Nowak, 1999; Michaelsen and Henderson, 2000; Diessel, 2007; Zdravkov et al., 2011). Many paleodepositional models of coal formation have been established in previous studies (Diessel, 1992; DiMichele and Phillips, 1994). The macerals, microlithotypes and lithotypes of coal can provide evidence about the nature and type of plant community, intensity and duration of humification and the type of depositional milieu (Singh and Singh P.K., 1996). Hence, quantitative relationships between the macerals and microlithotypes have been proposed and applied as useful methods of coal facies study. A ditrigonal coal facies diagram was proposed by Hacquebard and Donaldson (1969) and modified by Marchioni (1980). It has been suggested that the environment of coal deposition is controlled to a large extent by the water depth of the paleomires, which, in turn, controls the extent and nature of the floral development, the mode of plant debris preservation and the formation of different macerals and microlithotypes. Various types of coal facies under the terrestrial, telmatic, limno-telmatic and limnic settings are then identified with quantitative microlithotype parameters. The T-F-D concept, originally developed by Diessel (1982) and later prevailing in coal facies analysis (Marchioni and Kalkreuth, 1991; Obaje and Ligouis, 1996), defines the coal facies in terms of a ternary diagram, in which the end members are also parameters of microlithotypes. As methods of coal facies identification from maceral composition, the GI-TPI diagram (Diessel, 1986) and the GWI-VI diagram (Calder et al., 1991) are the most popular and have been applied to the coal formed under delta setting.

Research on the adsorption and desorption mechanism of CBM has been conducted for more than 50 years. Experimental studies and theoretical calculations of the adsorption and desorption behaviors of coal have been conducted with different gases, temperatures, pressures, humidity conditions, coal ranks, petrographic compositions and mathematical models (Daines, 1968; Joubert et al., 1973; Yang and Saunders, 1985; Day et al., 2008). In adsorption studies, single adsorbate, monolayer and polymolecular theories are the most common. The Langmuir equation (Langmuir, 1918) has been applied to the investigations of methane adsorption on coal as a typical monolayer adsorption theory (Zhou and Zhou L., 2009). Even though gas adsorption on coal is not monolayer adsorption, the Langmuir model is still applicable because the adsorption of methane on coal has a type I adsorption isotherm (Wang et al., 2012). The BET equation and D-A equation have been attached in the polymolecular layer and adsorption potential theory, respectively, of methane adsorption on coal. The applicability of various models to methane adsorption on coal and the mechanism interpretation in the light of the model parameters with physical meanings have also been extensively investigated.

The Gujiao Block in the Xishan Coalfield, Shanxi Province, North China, is an important commercial CBM production area in China, with estimated CBM resources of approximately 82 billion $\mathrm{m}^{3}$. The Xishan Lanyan CBM Corporation has drilled more than 700 CBM wells in the block since 2010, mainly targeting coal reservoirs No. 2 (Shanxi Formation), No. 8 and No. 9 (Taiyuan Formation) (Wang et al., 2015). The adsorption-desorption behaviors of coal have been shown to be critical to the productivity and economic benefit of CBM wells. The coal facies, as a comprehensive index of the matter composition and structure of coal, has significant influence on its adsorption-desorption characteristics and ultimately relates to the CBM drainage. In the present study, we conducted a series of analyses on the stratified channel samples collected from coal reservoirs No. 2, No. 4 and No. 9 in the Xiqu Drainage Area, Gujiao CBM Block. We expected (1) to distinguish the coal facies of the coal samples collected, (2) to describe the features and evolution of the paleomires, (3) to learn about the adsorption-desorption behaviors of coal in the light of some mathematical models and (4) to understand the relation between the coal facies and adsorption-desorption behaviors of the coal in the block. 


\section{Geological setting}

The Xiqu Drainage Area is located in the northern Gujiao CBM Block, central Shanxi Province, North China (Fig. 1). A series of NE-SW-striking normal faults of various scales occur in the area. The synclines and anticlines are of smaller scale and are parallel or perpendicular to the faults nearby. Coal-bearing strata in the block include the upper Carboniferous Benxi $\left(C_{2} b\right)$ and Taiyuan formations $\left(C_{2} t\right)$ and the lower Permian Shanxi $\left(P_{1} s\right)$ and Xiashihezi formations $\left(\mathrm{P}_{1} \mathrm{x}\right)$ (Fig. 2A).

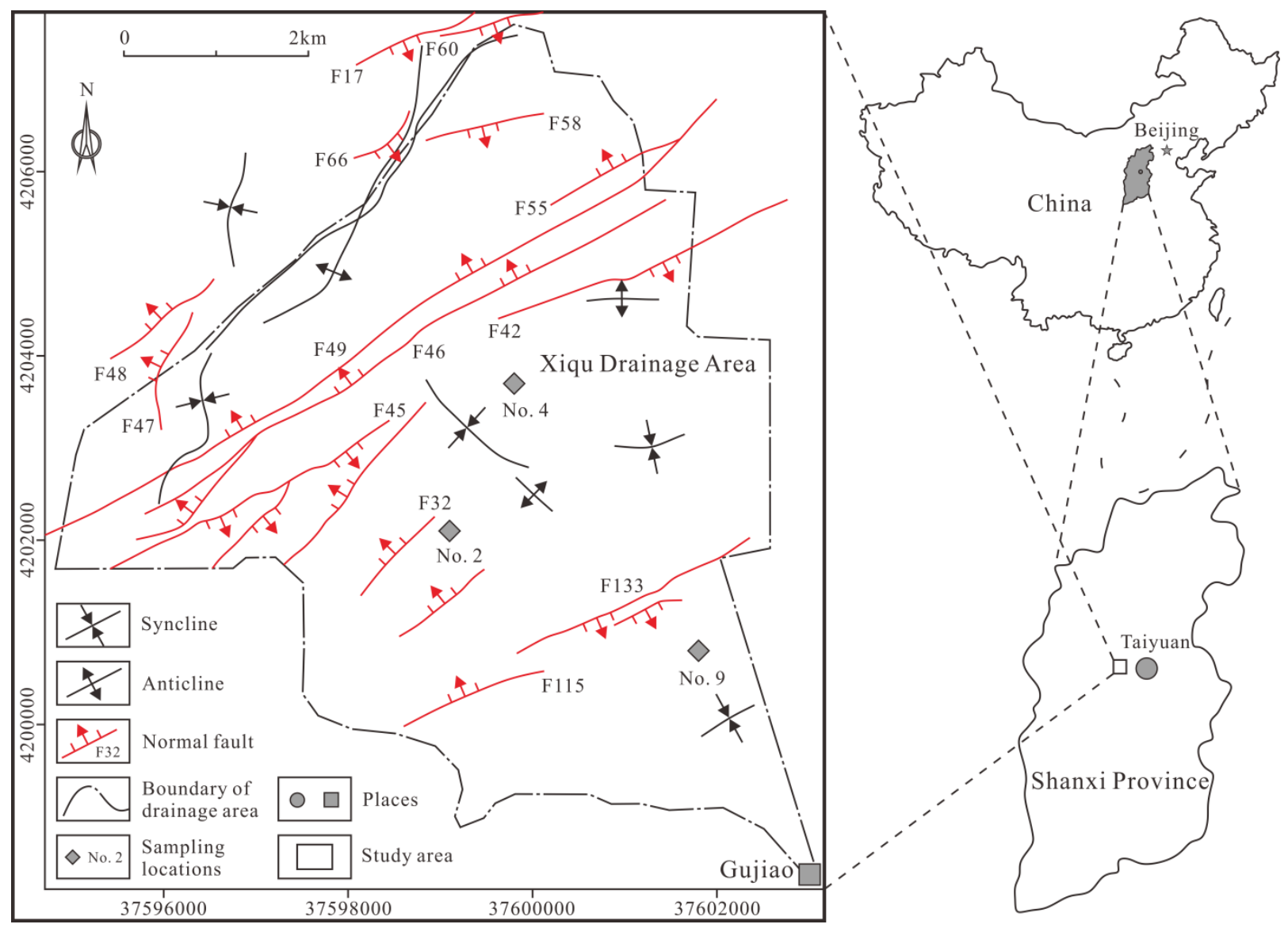

Fig. 1. Structure outline and sampling location map of the Xiqu Drainage Area.

The Taiyuan Formation, with thicknesses of $81.5 \sim 136.1 \mathrm{~m}$, conformably overlies the Benxi Formation and is mainly composed of sandstone, siltstone, mudstone, limestone and coal seams, in which coal seams No. 8 and No. 9 are two major CBM reservoirs. Coal seam No. 9, located in the lower part of the Taiyuan Formation, has a thickness of 0.52 2.86 m (average $1.65 \mathrm{~m}$ ) in the Xiqu Drainage Area. The Shanxi Formation, with thicknesses of 23.8 44.9 m, conformably overlies the Taiyuan Formation and is composed of sandstone, siltstone, mudstone and coal seams, in which coal seams No. 2 and No. 4 are another two major CBM reservoirs with thicknesses of 0.5 5.98 m (average $3.52 \mathrm{~m}$ ) and 0 4.09 $\mathrm{m}$ (average $2.12 \mathrm{~m}$ ), respectively. These major CBM reservoirs were formed in a marine-terrestrial transitional area and the peat mires developed mainly in the delta plain.

\section{Samples and methods}

\subsection{Sampling and preprocessing}

A total of 40 stratified channel samples were collected through vertical succession of the coal seams under the mine considering macrolithotypes such as bright coal, semibright coal, semidull coal and dull coal, following the 
procedures described in Sakorafa and Michailidis (1997), Taylor et al. (1998) and Kalkreuth et al. (2000). After freshing the non-weathered faces of the seams, coal seams No. 2, No. 4 and No. 9 were stratified into 15, 12 and 13 continuous parts, respectively, from the floors to the roofs, and every part, including the roofs and floors, was separately sampled (Fig. 2B). Unfortunately, a more detailed division of the coal seams, which would make for a more detailed coal facies study, could not be implemented owing to the limited sampling condition.

Coal samples were reduced incrementally and ground to a particle size of approximately $1 \mathrm{~mm}$ for polished sections, $0.4 \mathrm{~mm}$ for mercury porosimetry, $0.25 \mathrm{~mm}$ for isothermal adsorption-desorption experiment and $0.18 \mathrm{~mm}$ for proximate analysis and sulfur determination. Rock samples from the roof, floor and partings were prepared for sulfur determination.
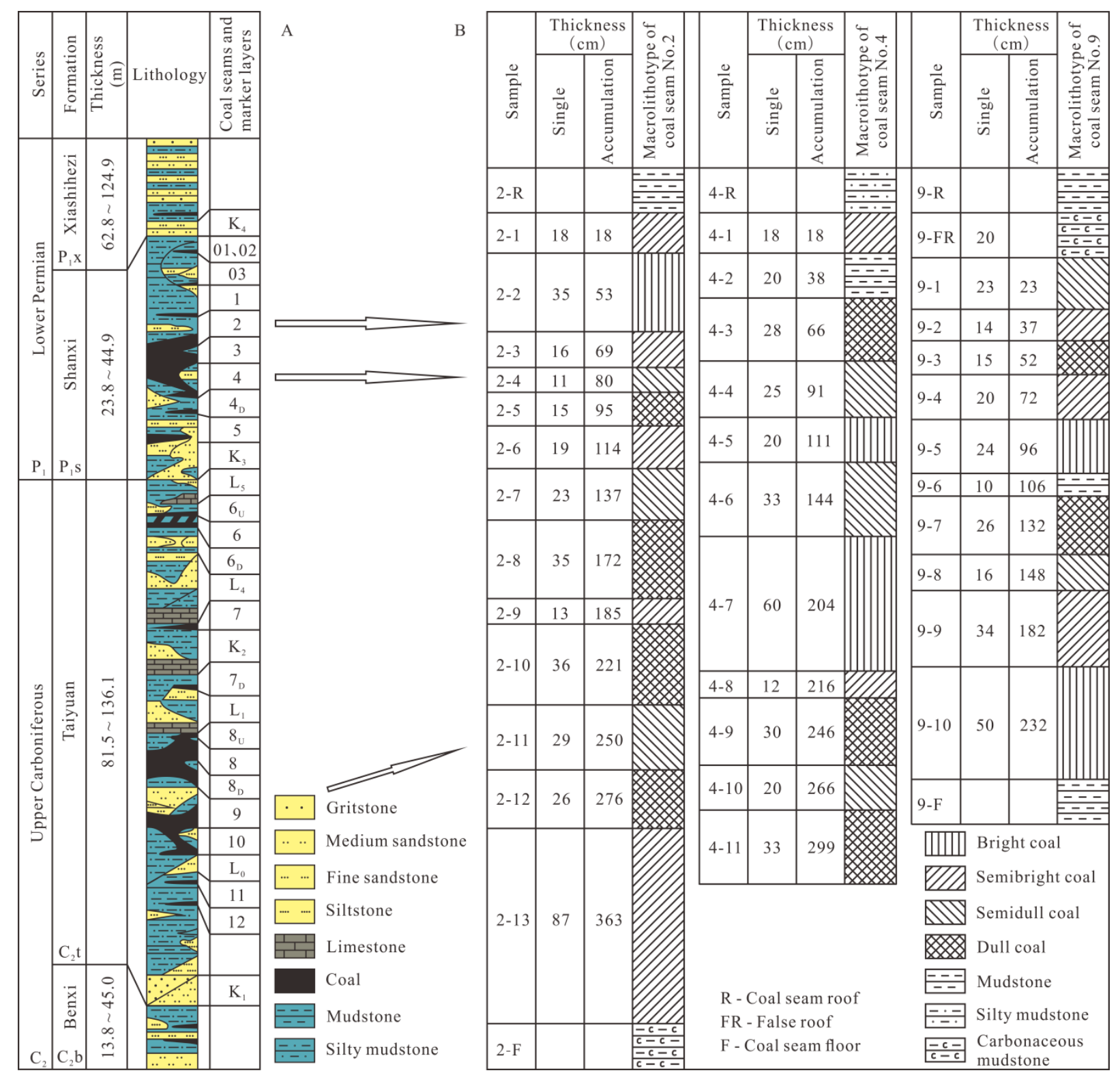

Fig. 2. Columnar sections in the Xiqu Drainage Area of the (A) Permo-Carboniferous coal-bearing strata and (B) samples.

\subsection{Micropetrographic analyses}

A fraction of the coal samples with $0.6 \sim 1.0 \mathrm{~mm}$ of granularity was used to prepare the polished sections, following the procedures established in and Taylor et al. (1998).

Vitrinite reflectance was measured with a CCD UVNIR microphotometer equipped with an Axio Imager and an M1m microscope, following the recommendations of Taylor et al. (1998). The examination was conducted using a 


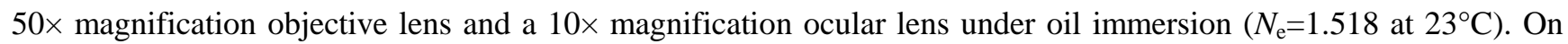
each sample at a wavelength of $546 \mathrm{~nm}$, maximum vitrinite (telocollinite) reflectance had fifty readings taken, by which mean maximum vitrinite reflectance was then calculated.

During maceral analysis, data of at least 500 points with a fixed step of $0.5 \mathrm{~mm}$ between each point were collected for each polished section, according to the method established by Taylor et al. (1998) and ISO 7404-3: 2009 (2009). Macerals were identified in white incident light as well as with blue irradiation, with a 50× magnification objective lens and a 10x magnification ocular lens under oil immersion using a Leitz Ortholux II POL-BK microscope.

\subsection{Isothermal adsorption-desorption experiments}

Following the procedures in Yao et al. (2009) and Xu et al. (2012), the Terra Tek ISO-300 was used for the isothermal adsorption-desorption experiments of 24 coal samples. The adsorption experiment was conducted from 0 $\mathrm{MPa}$ to approximately $10 \mathrm{MPa}$ at a constant temperature of $30^{\circ} \mathrm{C}$ (close to the actual reservoir temperature) on an air-dry basis. When equilibrium adsorption at the highest set pressure was reached, desorption experiments started at the same constant temperature. The residual gas content at every pressure point was recorded during depressurization until the amount of desorbed gas was less than $1.25 \times 10^{-2} \mathrm{~cm}^{3} / \mathrm{g}$ per day. Methane with an ultra-high purity (99.999\%) was used as the testing gas. Deviation of temperature during the course of the experiments was $\pm 0.5^{\circ} \mathrm{C}$. The accuracy of the pressure transducer was $\pm 0.1 \%$.

\subsection{Auxiliary analyses}

A series of auxiliary analyses were carried out. Moisture content, ash yield, volatile compounds and fixed carbon were determined with proximate analyzer SDTGA 5000. Mercury Porosimeter IV9500 with the pore diameter resolving power of $3 \mathrm{~nm}$ was used to measure the pore volume and specific surface area of coal samples. An Intelligence Sulfur Analyzer ZCS-8 was used to determine total sulfur content and occurring forms of sulfur.

\section{Results}

\subsection{Macroscopic appearance of the coals}

The studied coal seams were stratified on the basis of the macrolithotypes including the bright, semibright, semidull and dull coals (Fig. 2B). The bright and semibright coals are banded and have horizontal bedding, while dull coal appears relatively homogeneous and in massive structures, often associated with thin lutaceous interlayers. Coal seam No. 2 is predominantly composed of banded semibright coal, which constitutes $42.15 \%$ of the accumulated coal seam thickness, while bright coal is the least occurring type and accounts for merely 9.64\%. Bright and semibright coal dominate the upper and lower parts of coal seam No. 2, while the middle part contains mainly duller coal. Coal seam No. 4 is dominated with dull coal, which constitutes $32.62 \%$ of the total thickness of the coal seam. Semibright coal comprises only $10.75 \%$ of coal seam No. 4 and is the least-found macrolithotype in the seam. The upper and lower parts of coal seam No. 4 are predominantly dull and semidull coals; meanwhile, the middle part is brighter. Coal seam No. 9 is mainly dominated by bright and semibright coals from the floor to the roof, accounting for 33.33\% and $30.53 \%$, respectively, of the accumulated coal seam thickness. Dull coal is seen in the middle and upper parts of seam No. 9.

\subsection{Microscopic characteristic of the coals}

Vitrinite maximum reflectance $\left(R_{\mathrm{o}, \max }\right)$ ranges between $1.45 \%$ and $1.62 \%$ for coal seam No. 2 , between $1.56 \%$ 
and $1.70 \%$ for seam No. 4 and between $1.79 \%$ and $1.95 \%$ for seam No. 9 (Table 1), indicating that the former two seams rank coke coal and the last seam ranks meagre coal after the scheme established by Han et al. (1996), or all three seams rank bituminous A after the scheme established by ISO 11760: 2005 (2005). In general, there is a tendency of increasing vitrinite reflectance with the increasing depth of the studied coals.

Table 1. Data of vitrinite reflectance, coal quality and pore analyses of studied samples $\left(R_{0, \text { max }}\right.$, Proximate analysis and Sulfur analysis in $\% ; V_{\mathrm{m}}$ in $\mathrm{cm}^{3} / \mathrm{g}$; and $S_{\mathrm{m}}$ in $\mathrm{m}^{2} / \mathrm{g}$ ).

\begin{tabular}{|c|c|c|c|c|c|c|c|c|c|c|c|}
\hline \multirow{2}{*}{ Sample } & \multirow{2}{*}{$R_{\mathrm{o}, \max }$} & \multicolumn{4}{|c|}{ Proximate analysis } & \multicolumn{4}{|c|}{ Sulfur analysis } & \multirow{2}{*}{$V_{\mathrm{m}}$} & \multirow{2}{*}{$S_{\mathrm{m}}$} \\
\hline & & $M_{\mathrm{ad}}$ & $A_{\text {ad }}$ & $V_{\mathrm{ad}}$ & $F C_{\text {ad }}$ & $S_{\mathrm{p}, \mathrm{d}}$ & $S_{\mathrm{s}, \mathrm{d}}$ & $S_{\mathrm{o}, \mathrm{d}}$ & $S_{\mathrm{t}, \mathrm{d}}$ & & \\
\hline $2-\mathrm{R}$ & -- & -- & -- & -- & -- & 13.03 & 0.52 & 0.82 & 14.37 & -- & -- \\
\hline $2-1$ & 1.45 & 0.78 & 15.70 & 19.37 & 64.93 & 0.30 & 0.02 & 0.62 & 0.94 & 0.0175 & 14.462 \\
\hline $2-2$ & 1.57 & 0.83 & 18.64 & 21.25 & 60.12 & 0.10 & 0 & 0.50 & 0.60 & 0.0176 & 14.447 \\
\hline $2-3$ & 1.60 & 0.69 & 42.77 & 15.20 & 42.04 & 0.03 & 0 & 0.23 & 0.26 & 0.0073 & 6.093 \\
\hline $2-4$ & 1.55 & 0.86 & 24.85 & 18.62 & 56.54 & 0.06 & 0 & 0.40 & 0.46 & 0.0144 & 11.834 \\
\hline $2-5$ & 1.60 & 0.77 & 15.50 & 17.94 & 66.58 & 0.02 & 0 & 0.39 & 0.41 & 0.0177 & 14.614 \\
\hline $2-6$ & 1.56 & 0.78 & 13.61 & 20.76 & 65.63 & 0.03 & 0 & 0.48 & 0.51 & 0.0204 & 16.726 \\
\hline $2-7$ & 1.58 & 0.82 & 11.88 & 18.97 & 69.17 & 0.05 & 0.01 & 0.41 & 0.47 & 0.0199 & 16.401 \\
\hline $2-8$ & 1.62 & 0.80 & 17.26 & 17.25 & 65.51 & 0.26 & 0.03 & 0.46 & 0.75 & 0.0166 & 13.751 \\
\hline $2-9$ & 1.55 & 0.80 & 12.43 & 19.49 & 68.09 & 0.05 & 0.01 & 0.44 & 0.50 & 0.0206 & 17.000 \\
\hline $2-10$ & 1.55 & 0.81 & 8.17 & 19.72 & 72.12 & 0.03 & 0 & 0.43 & 0.46 & 0.0210 & 17.262 \\
\hline $2-11$ & 1.60 & 0.81 & 8.66 & 19.96 & 71.39 & 0.24 & 0.02 & 0.54 & 0.80 & 0.0220 & 18.084 \\
\hline $2-12$ & 1.55 & 0.85 & 8.26 & 19.80 & 71.95 & 0.10 & 0.01 & 0.49 & 0.60 & 0.0222 & 18.412 \\
\hline $2-13$ & 1.61 & 0.89 & 6.37 & 21.87 & 71.78 & 0.06 & 0.01 & 0.53 & 0.60 & 0.0218 & 17.870 \\
\hline $2-\mathrm{F}$ & -- & -- & -- & -- & -- & 2.37 & 0.14 & 0.29 & 2.80 & -- & -- \\
\hline $4-\mathrm{R}$ & -- & -- & -- & -- & -- & 0.44 & 0.08 & 0.06 & 0.58 & -- & -- \\
\hline 4-1 & 1.56 & 0.68 & 16.09 & 21.54 & 62.38 & 0.08 & 0.02 & 0.40 & 0.50 & 0.0176 & 14.483 \\
\hline $4-2$ & -- & -- & -- & -- & -- & 0.03 & 0 & 0.03 & 0.06 & -- & -- \\
\hline $4-3$ & 1.65 & 0.74 & 49.36 & 14.59 & 36.05 & 0.08 & 0.01 & 0.11 & 0.20 & 0.0062 & 5.188 \\
\hline $4-4$ & 1.61 & 0.72 & 30.56 & 15.99 & 53.46 & 0.11 & 0.02 & 0.31 & 0.44 & 0.0096 & 7.978 \\
\hline $4-5$ & 1.61 & 0.75 & 23.15 & 19.82 & 57.04 & 0.07 & 0.01 & 0.38 & 0.46 & 0.0140 & 11.488 \\
\hline $4-6$ & 1.62 & 0.91 & 16.02 & 18.59 & 65.39 & 0.10 & 0.02 & 0.39 & 0.51 & 0.0170 & 14.016 \\
\hline $4-7$ & 1.59 & 0.84 & 12.02 & 23.38 & 64.60 & 0.08 & 0.02 & 0.52 & 0.62 & 0.0178 & 14.614 \\
\hline $4-8$ & 1.63 & 0.83 & 11.67 & 20.32 & 68.01 & 0.08 & 0.02 & 0.44 & 0.54 & 0.0189 & 15.531 \\
\hline $4-9$ & 1.62 & 1.18 & 20.07 & 16.54 & 63.40 & 0.05 & 0.02 & 0.35 & 0.42 & 0.0142 & 11.754 \\
\hline $4-10$ & 1.61 & 0.79 & 17.82 & 18.82 & 63.37 & 0.03 & 0 & 0.39 & 0.42 & 0.0148 & 12.252 \\
\hline 4-11 & 1.70 & 0.95 & 27.65 & 16.38 & 55.98 & 0.05 & 0.01 & 0.29 & 0.35 & 0.0109 & 9.057 \\
\hline 9-R & -- & -- & -- & -- & -- & 0.82 & 0.07 & 0 & 0.89 & -- & -- \\
\hline 9-FR & -- & -- & -- & -- & -- & 10.96 & 0.94 & 0.73 & 12.63 & -- & -- \\
\hline $9-1$ & 1.95 & 1.75 & 8.14 & 14.72 & 77.15 & 0.95 & 0.14 & 0.59 & 1.68 & 0.0216 & 17.964 \\
\hline $9-2$ & 1.94 & 1.22 & 13.75 & 14.85 & 71.41 & 0.06 & 0.04 & 0.44 & 0.54 & 0.0202 & 16.684 \\
\hline $9-3$ & 1.90 & 0.87 & 35.26 & 13.69 & 51.06 & 0.06 & 0.02 & 0.20 & 0.28 & 0.0110 & 9.121 \\
\hline $9-4$ & 1.91 & 1.14 & 20.09 & 15.72 & 64.20 & 0.04 & 0.01 & 0.39 & 0.44 & 0.0147 & 12.084 \\
\hline $9-5$ & 1.79 & 1.14 & 14.33 & 16.87 & 68.81 & 0.20 & 0.02 & 0.51 & 0.73 & 0.0174 & 14.386 \\
\hline $9-6$ & -- & -- & -- & -- & -- & 0.03 & 0 & 0.07 & 0.10 & -- & -- \\
\hline $9-7$ & 1.89 & 0.98 & 27.13 & 13.56 & 59.32 & 0.03 & 0.01 & 0.26 & 0.30 & 0.0130 & 10.864 \\
\hline $9-8$ & 1.85 & 0.97 & 14.24 & 15.17 & 70.60 & 0.03 & 0.01 & 0.38 & 0.42 & 0.0177 & 14.613 \\
\hline $9-9$ & 1.91 & 1.02 & 11.79 & 16.29 & 71.93 & 0.03 & 0.02 & 0.41 & 0.46 & 0.0198 & 16.386 \\
\hline $9-10$ & 1.83 & 1.08 & 10.79 & 17.07 & 72.14 & 0.22 & 0.04 & 0.57 & 0.83 & 0.0206 & 16.887 \\
\hline $9-\mathrm{F}$ & -- & -- & -- & -- & -- & 0.02 & 0.02 & 0.04 & 0.08 & -- & -- \\
\hline
\end{tabular}


sulfur, dry basis; $V_{\mathrm{m}}$, volume of micropore; $S_{\mathrm{m}}$, specific surface area of micropore; R, coal seam roof; FR, false roof; F, coal seam floor;

Table 2. Petrographic composition of studied coal samples (vol\%).

\begin{tabular}{|c|c|c|c|c|c|c|c|c|c|c|c|c|c|c|c|c|c|}
\hline \multirow{2}{*}{ Sample } & \multicolumn{5}{|c|}{ Vitrinite group } & \multicolumn{6}{|c|}{ Inertinite group } & \multicolumn{3}{|c|}{ Liptinite group } & \multicolumn{3}{|c|}{ Mineral } \\
\hline & $\mathrm{T}$ & $\mathrm{TC}$ & DC & $\mathrm{CD}$ & $\sum \mathrm{V}$ & $\mathrm{F}$ & $\mathrm{Sf}$ & $\mathrm{Ma}$ & $\mathrm{Mi}$ & ID & $\sum \mathrm{I}$ & $\mathrm{MaS}$ & $\mathrm{Cu}$ & $\sum \mathrm{L}$ & Cla & Py & $\sum \mathrm{M}$ \\
\hline $2-1$ & 8.9 & 28.2 & 25.1 & 2.1 & 64.3 & 1.3 & 16.4 & 7.9 & 0.4 & 8.5 & 34.6 & 0.6 & 0 & 0.6 & 0 & 0.6 & 0.6 \\
\hline $2-2$ & 1.4 & 15.6 & 64.8 & 3.7 & 85.4 & 1.6 & 3.7 & 2.6 & 0.2 & 4.5 & 12.6 & 0.6 & 0.2 & 0.8 & 0.8 & 0.4 & 1.2 \\
\hline $2-3$ & 1.0 & 7.9 & 46.2 & 7.3 & 62.4 & 2.3 & 17.7 & 8.5 & 0 & 6.9 & 35.5 & 0 & 0.4 & 0.4 & 0.8 & 1.0 & 1.7 \\
\hline $2-4$ & 1.7 & 12.8 & 68.5 & 0.4 & 83.4 & 1.0 & 9.3 & 3.5 & 0 & 1.5 & 15.3 & 0.2 & 0 & 0.2 & 1.0 & 0.2 & 1.2 \\
\hline $2-5$ & 5.4 & 23.1 & 27.7 & 1.7 & 57.9 & 2.5 & 23.8 & 7.0 & 1.0 & 6.8 & 41.1 & 0.2 & 0 & 0.2 & 0.6 & 0.2 & 0.8 \\
\hline $2-6$ & 2.1 & 31.4 & 44.7 & 5.5 & 83.7 & 1.0 & 9.3 & 2.9 & 1.0 & 0.6 & 14.6 & 0.4 & 0.2 & 0.6 & 0.8 & 0.4 & 1.1 \\
\hline $2-7$ & 1.4 & 33.4 & 38.9 & 3.9 & 77.5 & 1.8 & 10.4 & 5.3 & 0.6 & 3.7 & 21.7 & 0.2 & 0 & 0.2 & 0.2 & 0.4 & 0.6 \\
\hline $2-8$ & 0.7 & 15.9 & 53.6 & 3.8 & 74.1 & 2.2 & 16.4 & 3.8 & 0.2 & 1.6 & 24.3 & 0 & 0.4 & 0.4 & 0.9 & 0.4 & 1.3 \\
\hline $2-9$ & 2.4 & 31.5 & 41.1 & 3.0 & 78.0 & 3.0 & 9.9 & 6.2 & 0.8 & 1.7 & 21.6 & 0.4 & 0 & 0.4 & 0 & 0 & 0 \\
\hline $2-10$ & 9.5 & 41.8 & 19.9 & 3.0 & 74.2 & 2.6 & 18.6 & 2.2 & 0.2 & 1.3 & 24.9 & 0.4 & 0 & 0.4 & 0.2 & 0.4 & 0.6 \\
\hline $2-11$ & 7.8 & 32.1 & 23.7 & 4.3 & 67.9 & 1.8 & 15.3 & 7.0 & 0.6 & 6.7 & 31.3 & 0.4 & 0 & 0.4 & 0 & 0.4 & 0.4 \\
\hline $2-12$ & 5.8 & 43.5 & 24.3 & 1.3 & 74.9 & 1.9 & 16.9 & 2.4 & 0.6 & 2.0 & 23.8 & 0 & 0.6 & 0.6 & 0.4 & 0.4 & 0.7 \\
\hline $2-13$ & 6.3 & 36.4 & 34.3 & 2.1 & 79.0 & 3.0 & 10.7 & 2.9 & 0 & 3.6 & 20.2 & 0.2 & 0 & 0.2 & 0.4 & 0.2 & 0.6 \\
\hline 4-1 & 6.5 & 36.4 & 39.9 & 1.3 & 84.1 & 0.9 & 11.1 & 2.4 & 0 & 1.1 & 15.5 & 0.4 & 0 & 0.4 & 0 & 0 & 0 \\
\hline 4-3 & 0.6 & 6.4 & 19.9 & 7.4 & 34.3 & 2.5 & 23.8 & 23.2 & 0 & 13.8 & 63.4 & 0.8 & 0 & 0.8 & 1.2 & 0.4 & 1.6 \\
\hline 4-4 & 0.8 & 21.9 & 41.6 & 3.8 & 68.1 & 1.0 & 19.8 & 6.5 & 0 & 3.2 & 30.4 & 0.4 & 0 & 0.4 & 0.6 & 0.6 & 1.1 \\
\hline $4-5$ & 3.7 & 33.3 & 43.6 & 2.7 & 83.3 & 1.4 & 9.9 & 2.7 & 0 & 1.4 & 15.4 & 0 & 0.4 & 0.4 & 0.6 & 0.4 & 1.0 \\
\hline 4-6 & 2.7 & 40.6 & 10.7 & 4.1 & 58.1 & 3.7 & 27.8 & 3.7 & 0 & 5.4 & 40.6 & 0 & 0.6 & 0.6 & 0.4 & 0.4 & 0.8 \\
\hline $4-7$ & 5.2 & 36.5 & 35.5 & 2.3 & 79.5 & 1.4 & 12.2 & 4.8 & 0 & 1.2 & 19.5 & 0.4 & 0 & 0.4 & 0.2 & 0.4 & 0.6 \\
\hline 4-8 & 6.4 & 39.3 & 20.4 & 1.5 & 67.6 & 1.7 & 19.7 & 8.8 & 0 & 1.9 & 32.0 & 0 & 0.4 & 0.4 & 0 & 0 & 0 \\
\hline 4-9 & 1.9 & 42.7 & 28.5 & 5.9 & 78.9 & 1.1 & 7.8 & 1.7 & 0 & 9.3 & 19.9 & 0 & 0.4 & 0.4 & 0.6 & 0.2 & 0.8 \\
\hline 4-10 & 5.7 & 31.7 & 23.2 & 6.7 & 67.3 & 2.6 & 17.7 & 5.7 & 0 & 5.9 & 31.9 & 0.4 & 0 & 0.4 & 0 & 0.4 & 0.4 \\
\hline 4-11 & 0.6 & 21.1 & 43.0 & 7.0 & 71.7 & 1.1 & 18.4 & 4.6 & 0 & 3.0 & 27.2 & 0.4 & 0 & 0.4 & 0.4 & 0.4 & 0.8 \\
\hline $9-1$ & 4.6 & 24.0 & 24.2 & 2.6 & 55.4 & 2.0 & 26.8 & 7.6 & 0.4 & 6.5 & 43.3 & 0 & 0 & 0 & 0.4 & 0.9 & 1.3 \\
\hline $9-2$ & 1.4 & 35.5 & 32.6 & 8.0 & 77.5 & 1.0 & 12.3 & 3.3 & 0.8 & 3.7 & 21.1 & 0.6 & 0 & 0.6 & 0.6 & 0.2 & 0.8 \\
\hline $9-3$ & 2.1 & 6.2 & 20.6 & 6.0 & 35.0 & 3.5 & 40.6 & 16.9 & 0 & 1.9 & 62.9 & 0.8 & 0 & 0.8 & 0.8 & 0.6 & 1.4 \\
\hline $9-4$ & 4.8 & 38.5 & 26.2 & 7.1 & 76.6 & 0.8 & 8.2 & 9.6 & 1.1 & 2.9 & 22.6 & 0.4 & 0 & 0.4 & 0.4 & 0 & 0.4 \\
\hline $9-5$ & 4.3 & 41.3 & 30.2 & 7.6 & 83.3 & 0.6 & 7.4 & 6.2 & 0 & 1.7 & 15.9 & 0.4 & 0 & 0.4 & 0.4 & 0 & 0.4 \\
\hline $9-7$ & 1.3 & 18.3 & 50.4 & 4.9 & 74.9 & 1.3 & 14.9 & 4.5 & 0.2 & 2.6 & 23.6 & 0 & 0.4 & 0.4 & 0.8 & 0.4 & 1.1 \\
\hline $9-8$ & 4.0 & 28.4 & 20.5 & 1.7 & 54.6 & 1.7 & 26.4 & 12.1 & 0 & 4.1 & 44.3 & 0 & 0.4 & 0.4 & 0.6 & 0.2 & 0.8 \\
\hline $9-9$ & 4.5 & 40.6 & 31.5 & 5.8 & 82.4 & 1.1 & 8.7 & 5.8 & 1.1 & 0.4 & 17.1 & 0 & 0 & 0 & 0.6 & 0 & 0.6 \\
\hline $9-10$ & 7.4 & 54.4 & 26.5 & 4.6 & 93.0 & 1.7 & 3.5 & 0.7 & 0 & 0.4 & 6.3 & 0 & 0 & 0 & 0.4 & 0.4 & 0.7 \\
\hline
\end{tabular}

$\mathrm{T}$, telinite; TC, telocollinite; $\mathrm{DC}$, desmocollinite; $\mathrm{CD}$, vitrodetrinite; $\mathrm{V}$, vitrinite group; $\mathrm{F}$, fusinite; Sf, semifusinite; Ma, macrinite; Mi, micrinite; ID, inertodetrinite; I, inertinite group; MaS, macrosporinite; $\mathrm{Cu}$, cutinite; L, liptinite group; Cla, clay mineral; Py, pyrite; M, mineral matter.

A total of 11 macerals were counted in all coal samples and the vitrinite group is at an advantage in the macerals (Table 2). Vitrinite group in coal seam No. 2 accounts for 57.9 85.4 vol\% of the overall composition. Comparatively, vitrinite group macerals vary by a broader margin in other seams, with $34.3 \sim 84.1$ vol\% found in coal seam No. 4 and 35.0 93.0 vol\% found in coal seam No. 9. In this group, the predominant macerals are telocollinite and desmocollinite while telinite and vitrodetrinite are found in small amounts. The inertinite group is less abundant, accounting for 12.6 41.1 vol\%, 15.4 63.4 vol\% and 6.3 62.9 vol\% in coal seams No. 2, No. 4 and No. 9, respectively, and is mainly composed of semifusinite. Macrinite occurrence is characterized by the broadest distribution, ranging between 0.7 23.2 vol\%. A small amount of elliptical micrinite is found in the inertinite group and is often associated with telocollinite. The liptinite group is rare and constitutes less than 0.8 vol\% of the overall 
composition. Only macrosporinite and cutinite are observed. The highest content of visible mineral matter is 1.7 vol\%, and only clay mineral and pyrite are found. Pyrite occurring as small, euhedral crystals or in framboidal form is often associated with desmocollinite. This type of pyrite is usually interpreted as syngenetic pyrite during the peat formation (Taylor et al., 1998). Epigenetic pyrite is present as filling of open cell lumens in some macerals such as telocollinite and semifusinite.

\subsection{Isothermal adsorption-desorption characteristics}

Adsorption and desorption experiments on 24 coal samples were conducted successively at a constant temperature of $30^{\circ} \mathrm{C}$. The results are listed in Table 3, Table 4 and Fig. 3. The maximum adsorbed gas content $\left(V_{\max }\right)$ was measured when equilibrium adsorption was reached at the highest set pressure of approximately $10 \mathrm{MPa}$. $V_{\max }$ of coal seams No. 2, No. 4 and No. 9 range between $7.34 \sim 19.35 \mathrm{~cm}^{3} / \mathrm{g}$ (mean $15.16 \mathrm{~cm}^{3} / \mathrm{g}$ ), $6.86 \sim 17.00 \mathrm{~cm}^{3} / \mathrm{g}$ (mean $11.47 \mathrm{~cm}^{3} / \mathrm{g}$ ) and $10.41 \sim 20.86 \mathrm{~cm}^{3} / \mathrm{g}$ (mean $16.75 \mathrm{~cm}^{3} / \mathrm{g}$ ), respectively.

Table 3. Isothermal adsorption data of studied coal samples ( $p$ in MPa and $V_{\mathrm{ad}}$ in $\mathrm{cm}^{3} / \mathrm{g}$ ).

\begin{tabular}{|c|c|c|c|c|c|c|c|c|c|c|c|c|c|c|c|}
\hline \multicolumn{2}{|c|}{$2-1$} & \multicolumn{2}{|c|}{$2-2$} & \multicolumn{2}{|c|}{$2-3$} & \multicolumn{2}{|c|}{$2-5$} & \multicolumn{2}{|c|}{$2-7$} & \multicolumn{2}{|c|}{$2-10$} & \multicolumn{2}{|c|}{ 2-12 } & \multicolumn{2}{|c|}{$2-13$} \\
\hline$p$ & $V_{\text {ad }}$ & $p$ & $V_{\text {ad }}$ & $p$ & $V_{\text {ad }}$ & $p$ & $V_{\text {ad }}$ & $p$ & $V_{\mathrm{ad}}$ & $p$ & $V_{\mathrm{ad}}$ & $p$ & $V_{\mathrm{ad}}$ & $p$ & $V_{\mathrm{ad}}$ \\
\hline 0 & 0 & 0 & 0 & 0 & 0 & 0 & 0 & 0 & 0 & 0 & 0 & 0 & 0 & 0 & \\
\hline 0.56 & 4.74 & 0.68 & 3.32 & 0.71 & 1.45 & 0.66 & 3.53 & 0.67 & 3.14 & 0.53 & 4.79 & 0.56 & 3.90 & 0.58 & 4.11 \\
\hline 2.40 & 12.03 & 2.67 & 8.24 & 2.89 & 4.07 & 2.64 & 8.80 & 2.64 & 8.79 & 2.37 & 12.50 & 2.47 & 10.29 & 2.27 & 10.55 \\
\hline 4.48 & 15.04 & 4.78 & 10.24 & 5.03 & 5.31 & 4.75 & 11.17 & 4.61 & 11.40 & 4.52 & 15.67 & 4.88 & 13.31 & 4.89 & 14.39 \\
\hline 6.57 & 17.23 & 6.65 & 11.11 & 6.86 & 6.25 & 6.58 & 12.56 & 6.52 & 13.15 & 6.52 & 17.43 & 6.60 & 14.63 & 6.65 & 16.02 \\
\hline 8.38 & 18.34 & 8.30 & 11.55 & 8.12 & 6.66 & 8.25 & 13.33 & 8.35 & 14.34 & 8.28 & 18.38 & 8.06 & 15.39 & 8.19 & 17.00 \\
\hline 10.33 & 19.35 & .24 & 11.92 & 10.67 & 7.34 & 10.33 & 13.87 & 10.35 & 15.33 & 0.32 & 19.25 & 10.39 & & 10.40 & 17.85 \\
\hline \multicolumn{2}{|c|}{$4-3$} & \multicolumn{2}{|c|}{ 4-4 } & \multicolumn{2}{|c|}{$4-5$} & \multicolumn{2}{|c|}{ 4-6 } & \multicolumn{2}{|c|}{ 4-7 } & \multicolumn{2}{|c|}{ 4-9 } & \multicolumn{2}{|c|}{ 4-11 } & \multicolumn{2}{|c|}{$9-1$} \\
\hline$p$ & $V_{\text {ad }}$ & $p$ & $V_{\mathrm{ad}}$ & $p$ & $V_{\text {ad }}$ & $p$ & $V_{\text {ad }}$ & $p$ & $V_{\text {ad }}$ & $p$ & $V_{\text {ad }}$ & $p$ & $V_{\text {ad }}$ & $p$ & $V_{\text {ad }}$ \\
\hline 0 & 0 & 0 & 0 & 0 & 0 & 0 & 0 & 0 & 0 & 0 & 0 & 0 & 0 & 0 & \\
\hline 0.84 & 1.93 & 0.81 & 1.55 & 0.69 & 2.60 & 0.58 & 3.80 & 0.59 & 3.42 & 0.75 & 2.43 & 0.72 & 2.21 & 0.56 & 4.24 \\
\hline 2.88 & 4.22 & 2.91 & 4.27 & 2.75 & 6.75 & 2.67 & 9.99 & 2.64 & 9.37 & 2.82 & 6.36 & 2.94 & 6.07 & 2.33 & 11.35 \\
\hline 4.94 & 5.20 & 4.97 & 5.64 & 4.88 & 5 & 4.66 & 13.17 & 4.63 & 11.73 & 4.81 & 8.08 & 4.80 & 7.81 & 4.86 & 14.8 \\
\hline 6.83 & 6.16 & 6.57 & 6.78 & 6.66 & 9.05 & 6.51 & 14.92 & 6.52 & 13.20 & 6.45 & 9.42 & 6.72 & 9.61 & 6.57 & 16.31 \\
\hline 8.54 & 6.58 & 7.98 & 7.33 & 8.54 & 10.57 & 8.11 & 16.05 & 8.07 & 13.94 & 8.36 & 10.22 & 8.25 & 10.64 & 8.30 & 17.30 \\
\hline 10.49 & 6.86 & 10.56 & 8.11 & 10.59 & 11.31 & 11.18 & 17.00 & 10.33 & 14.51 & 10.36 & 10.80 & 11.26 & 11.72 & 10.31 & 18.32 \\
\hline \multicolumn{2}{|c|}{$9-2$} & \multicolumn{2}{|c|}{$9-3$} & \multicolumn{2}{|c|}{$9-4$} & \multicolumn{2}{|c|}{$9-5$} & \multicolumn{2}{|c|}{$9-7$} & \multicolumn{2}{|c|}{$9-8$} & \multicolumn{2}{|c|}{$9-9$} & \multicolumn{2}{|c|}{$9-10$} \\
\hline$p$ & $V_{\text {ad }}$ & $p$ & $V_{\text {ad }}$ & $p$ & $V_{\text {ad }}$ & $p$ & $V_{\text {ad }}$ & $p$ & $V_{\text {ad }}$ & $p$ & $V_{\mathrm{ad}}$ & $p$ & $V_{\mathrm{ad}}$ & $p$ & $V_{\text {ad }}$ \\
\hline 0 & 0 & 0 & 0 & 0 & 0 & 0 & 0 & 0 & 0 & 0 & 0 & 0 & 0 & 0 & \\
\hline 0.56 & 3.05 & 0.68 & 2.88 & 0.65 & 3.20 & 0.62 & 3.56 & 0.72 & 3.08 & 0.43 & 4.08 & 0.50 & 4.67 & 0.61 & 4.40 \\
\hline 2.54 & 9.46 & 2.70 & 6.89 & 2.63 & 8.95 & 2.54 & 10.12 & 2.70 & 7.68 & 2.29 & 13.00 & 2.43 & 13.32 & 2.51 & 12.35 \\
\hline 4.67 & 12.29 & 4.84 & 8.48 & 4.62 & 11.42 & 4.81 & 13.47 & 4.97 & 10.39 & 4.27 & 16.20 & 4.60 & 16.56 & 4.65 & 15.73 \\
\hline 6.69 & 13.96 & 6.66 & 9.39 & 6.61 & 12.91 & 6.64 & 15.21 & 6.38 & 11.82 & 6.40 & 18.52 & 6.64 & 18.86 & 6.66 & 17.78 \\
\hline 8.02 & 14.60 & 8.30 & 9.86 & 8.36 & 13.87 & 8.29 & 16.42 & 7.94 & 12.82 & 8.25 & 19.68 & 8.17 & 19.73 & 8.47 & 18.82 \\
\hline 10.70 & 15.72 & 10.6 & 10.41 & 10.33 & 14.83 & 10.40 & 17.20 & 10.54 & 13.70 & 10.37 & 20.86 & 11.18 & 20.28 & 10.64 & 19.39 \\
\hline
\end{tabular}

$p$, pressure; $V_{\text {ad }}$, adsorbed gas content.

For a better understanding of the isothermal adsorption behavior, Langmuir equation (1) (Langmuir, 1918), BET equation with two constants (2) and D-A equation (3), listed below, were used as representative adsorption models of monolayer, polymolecular layer and adsorption potential theory, respectively.

$$
V=V_{L} \frac{p}{p_{L}+p}
$$


218 where $V$ is the gas content within the sample, $\mathrm{cm}^{3} / \mathrm{g} ; V_{\mathrm{L}}$ is Langmuir volume, $\mathrm{cm}^{3} / \mathrm{g} ; p$ is pressure, $\mathrm{MPa}$; and $p_{\mathrm{L}}$ is

219 Langmuir pressure, MPa.

$$
V=\frac{V_{m} C p}{\left(p^{0}-p\right)\left[1+(C-1) \frac{p}{p^{0}}\right]}
$$

220 where $V_{\mathrm{m}}$ is the maximum monolayer gas content within the sample, $\mathrm{cm}^{3} / \mathrm{g} ; C$ is constant relevant to adsorption heat;

221 and $p^{0}$ is saturated vapor pressure, MPa.

$$
V=V_{0} \exp \left[-D \ln ^{n}\left(\frac{p^{0}}{p}\right)\right]
$$

222 where $V_{0}$ is the volume of micropore, $\mathrm{cm}^{3} / \mathrm{g} ; D$ is constant relevant to adsorption heat; and $n$ is constant relevant to 223 pore distribution.

\begin{tabular}{|c|c|c|c|c|c|c|c|c|c|c|c|c|c|c|c|}
\hline \multicolumn{2}{|c|}{$2-1$} & \multicolumn{2}{|c|}{$2-2$} & \multicolumn{2}{|c|}{$2-3$} & \multicolumn{2}{|c|}{$2-5$} & \multicolumn{2}{|c|}{$2-7$} & \multicolumn{2}{|c|}{$2-10$} & \multicolumn{2}{|c|}{$2-12$} & \multicolumn{2}{|c|}{$2-13$} \\
\hline$p$ & $V_{\mathrm{r}, \text { ad }}$ & $p$ & $V_{\mathrm{r}, \text { ad }}$ & $p$ & $V_{\mathrm{r}, \text { ad }}$ & $p$ & $V_{\mathrm{r}, \mathrm{ad}}$ & $p$ & $V_{\mathrm{r}, \mathrm{ad}}$ & $p$ & $V_{\mathrm{r}, \text { ad }}$ & $p$ & $V_{\mathrm{r}, \text { ad }}$ & $p$ & $V_{\mathrm{r}, \text { ad }}$ \\
\hline 8.44 & 19.24 & 8.04 & 11.81 & 8.31 & 7.21 & 7.87 & 13.76 & 8.02 & 14.96 & 8.56 & 19.28 & 8.21 & 15.99 & 7.59 & 17.53 \\
\hline 6.99 & 18.38 & 6.76 & 11.59 & 6.98 & 6.76 & 7.03 & 13.25 & 6.60 & 13.86 & 7.10 & 18.53 & 7.08 & 15.30 & 6.93 & 17.03 \\
\hline 5.55 & 17.40 & 5.57 & 11.16 & 5.87 & 6.38 & 6.11 & 12.77 & 5.51 & 13.03 & 5.70 & 17.65 & 5.95 & 14.53 & 6.12 & 16.46 \\
\hline 5.08 & 17.02 & 4.67 & 10.63 & 4.94 & 5.95 & 4.88 & 11.91 & 4.42 & 12.03 & 5.07 & 17.15 & 5.25 & 13.97 & 5.01 & 15.47 \\
\hline 3.77 & 15.84 & 3.63 & 9.85 & 3.96 & 5.47 & 3.80 & 11.01 & 3.45 & 10.97 & 3.82 & 16.01 & 3.71 & 12.49 & 3.67 & 13.99 \\
\hline 2.76 & 14.53 & 2.66 & 8.84 & 2.83 & 4.80 & 2.84 & 10.01 & 2.51 & 9.73 & 2.84 & 14.75 & 2.40 & 10.69 & 2.83 & 12.78 \\
\hline 1.93 & 13.11 & 1.72 & 7.37 & 1.88 & 4.06 & 1.95 & 8.74 & 1.63 & 8.07 & 2.01 & 13.33 & 1.65 & 9.10 & 2.00 & 11.24 \\
\hline 1.22 & 11.24 & 1.09 & 5.92 & 1.25 & 3.51 & 1.18 & 7.24 & 1.06 & 6.66 & 1.31 & 11.51 & 1.30 & 8.22 & 1.27 & 9.43 \\
\hline 0.82 & 9.74 & 0.71 & 4.62 & 0.69 & 266 & 0.77 & 5 & 0.64 & 5.02 & 0.90 & 10.02 & 0.88 & 8 & 0.86 & 7.91 \\
\hline \multicolumn{2}{|c|}{ 4-3 } & \multicolumn{2}{|c|}{$4-4$} & \multicolumn{2}{|c|}{$4-5$} & \multicolumn{2}{|c|}{$4-6$} & \multicolumn{2}{|c|}{ 4-7 } & \multicolumn{2}{|c|}{$4-9$} & \multicolumn{2}{|c|}{ 4-11 } & \multicolumn{2}{|c|}{$9-1$} \\
\hline$p$ & $V_{\mathrm{r}, \mathrm{ad}}$ & $p$ & $V_{\mathrm{r}, \text { ad }}$ & $p$ & $V_{\mathrm{r}, \mathrm{ad}}$ & $p$ & $\mathrm{~V}_{\mathrm{r}, \mathrm{ad}}$ & $p$ & $V_{\mathrm{r}, \mathrm{ad}}$ & $p$ & $V_{\mathrm{r}, \text { ad }}$ & $p$ & $V_{\mathrm{r}, \mathrm{ad}}$ & $p$ & $V_{\mathrm{r}, \text { ad }}$ \\
\hline 8.61 & 6.82 & 8.11 & 8.10 & 8.29 & 10.90 & 8.43 & 16.67 & 7.81 & 14.37 & 8.06 & 10.73 & 8.43 & 11.53 & 8.20 & 17.98 \\
\hline 7.05 & 6.52 & 7.04 & 7.75 & 7.14 & 10.35 & 6.64 & 15.62 & 7.02 & 13.94 & 7.17 & 10.32 & 6.75 & 10.88 & 7.13 & 17.30 \\
\hline 5.75 & 6.21 & 5.92 & 7.38 & 6.03 & 9.80 & 5.45 & 14.74 & 6.16 & 13.44 & 6.23 & 9.95 & 5.48 & 10.28 & 6.07 & 16.57 \\
\hline 5.07 & 6.03 & 4.86 & 6.88 & 5.29 & 9.33 & 4.51 & 13.99 & 4.88 & 12.50 & 5.05 & 9.27 & 4.57 & 9.76 & 5.37 & 16.00 \\
\hline 3.75 & 5.67 & 3.85 & & 3.84 & & 3.52 & 13 & 3.78 & 11 & 3.74 & 8.45 & 3.56 & 9.23 & 3.75 & 14.38 \\
\hline 2.72 & 5.21 & 2.83 & 5.72 & 2.59 & 7.25 & 2.42 & 11.71 & 2.57 & 10.12 & 2.83 & 7.75 & 2.59 & 8.41 & 2.57 & 12.70 \\
\hline 1.86 & 4.76 & 1.88 & 4.93 & 1.57 & 5.86 & 1.74 & 10.61 & 1.86 & 8.89 & 1.96 & 6.77 & 1.78 & 7.67 & 1.61 & 10.62 \\
\hline 1.11 & 4.03 & 1.19 & 4.27 & 1.19 & 5.22 & 1.06 & 8.85 & 1.17 & 7.47 & 1.17 & 5.75 & 1.05 & 6.26 & 1.15 & 9.27 \\
\hline 0.69 & 3.52 & 0.62 & 3.29 & 0.92 & 4.52 & 0.67 & 7.48 & 0.79 & 6.21 & 0.76 & 4.75 & 0.63 & 5.38 & 0.82 & 7.88 \\
\hline \multicolumn{2}{|c|}{$9-2$} & \multicolumn{2}{|c|}{$9-3$} & \multicolumn{2}{|c|}{$9-4$} & \multicolumn{2}{|c|}{ 9-5 } & \multicolumn{2}{|c|}{$9-7$} & \multicolumn{2}{|c|}{$9-8$} & \multicolumn{2}{|c|}{$9-9$} & \multicolumn{2}{|c|}{$9-10$} \\
\hline$p$ & $V_{\mathrm{r}, \mathrm{ad}}$ & $p$ & $V_{\mathrm{r}, \text { ad }}$ & $p$ & $V_{\mathrm{r}, \mathrm{ad}}$ & $p$ & $\mathrm{~V}_{\mathrm{r}, \mathrm{ad}}$ & $p$ & $V_{\mathrm{r}, \mathrm{ad}}$ & $p$ & $V_{\mathrm{r}, \text { ad }}$ & $p$ & $V_{\mathrm{r}, \mathrm{ad}}$ & $p$ & $V_{\mathrm{r}, \text { ad }}$ \\
\hline 8.12 & 15.69 & 8.20 & 10.20 & 7.87 & 14.39 & 7.79 & 17.08 & 8.03 & 13.58 & 8.20 & 20.82 & 8.49 & 20.14 & 8.61 & 19.06 \\
\hline 6.80 & 14.90 & 7.03 & 9.71 & 6.56 & 13.50 & 6.45 & 15.90 & 6.81 & 13.20 & 6.95 & 20.05 & 6.45 & 18.83 & 7.39 & 18.39 \\
\hline 5.55 & 14.16 & 5.84 & 9.26 & 5.54 & 12.83 & 5.25 & 14.87 & 5.67 & 12.64 & 5.56 & 19.09 & 5.03 & 17.60 & 6.08 & 17.53 \\
\hline 4.56 & 13.35 & 5.18 & 8.93 & 4.44 & 11.91 & 4.38 & 13.92 & 4.60 & 12.14 & 5.06 & 18.68 & 4.31 & 16.86 & 5.18 & 16.91 \\
\hline 3.55 & 12.30 & 3.93 & 8.24 & 3.38 & 10.82 & 3.33 & 12.60 & 3.43 & 11.20 & 3.77 & 17.47 & 3.43 & 15.88 & 4.20 & 15.95 \\
\hline 2.55 & 10.91 & 2.68 & 7.31 & 2.49 & 9.64 & 2.41 & 11.15 & 2.50 & 10.13 & 2.86 & 16.25 & 2.42 & 14.23 & 2.94 & 14.89 \\
\hline 1.64 & 9.20 & 1.67 & 6.13 & 1.63 & 8.00 & 1.56 & 9.21 & 1.58 & 8.80 & 2.03 & 14.84 & 1.77 & 12.88 & 1.98 & 13.19 \\
\hline 1.08 & 7.65 & 1.08 & 5.17 & 1.07 & 6.57 & 0.91 & 7.23 & 1.00 & 7.73 & 1.32 & 12.98 & 1.10 & 10.73 & 1.38 & 11.52 \\
\hline 0.66 & 5.91 & 0.70 & 4.19 & 0.66 & 4.94 & 0.58 & 5.57 & 0.59 & 6.48 & 0.90 & 11.46 & 0.73 & 9.03 & 0.92 & 9.70 \\
\hline
\end{tabular}

Table 4. Isothermal desorption data of studied coal samples ( $p$ in MPa and $V_{\mathrm{r} \text {, ad }}$ in $\mathrm{cm}^{3} / \mathrm{g}$ ). 
Fig. 3. Adsorption and desorption isotherms of studied coal samples. Note: ad., adsorption; de., desorption.

Matlab R2010b was used and parameters of equations (1), (2) and (3) were obtained using nonlinear fitting solvers at least squares principle. Convergence cannot be obtained for equation (2). Valid parameters for equations (1) and (3) are listed in Table 5.

The Langmuir equation can also be used to evaluate desorption experiments and $V_{\mathrm{L}}$ and $p_{\mathrm{L}}$ for the desorption experiments are marked as $V_{\mathrm{L}, \mathrm{D}}$ and $p_{\mathrm{L}, \mathrm{D}}$ in present study(Table 5). Depletion gas content ( $\left.V_{\mathrm{d}}\right)$ was calculated using the assumed depletion pressure of $0.7 \mathrm{MPa}$, an empirical value of the lowest reservoir pressure obtained during CBM drainage in America (Fu et al., 2007). Eventually, desorption rate $\left(R_{\mathrm{D}}\right)$ was worked out while the desorbed gas content $\left(V_{\mathrm{D}}\right)$ was divided by the maximum adsorbed gas content $\left(V_{\max }\right)$ (Table 5).

Both the $V_{\mathrm{L}}$ and $V_{0}$ correlate positively with $V_{\max }$ (Fig. 4A), indicating the ultimate adsorption capacity of coal seam No. 9 is the highest and that of coal seam No. 4 is the lowest. Similarly, the $V_{\mathrm{d}}$ positively correlates with $V_{\text {max }}$ (Fig. 4A), meaning the higher the adsorbed gas content is in the adsorption, the higher the residual gas content will be after desorption. $V_{\mathrm{L}, \mathrm{D}}$ is directly proportional to and consistently lower than $V_{\mathrm{L}}$ (Fig. 4B). Positive correlations also occur between $p_{\mathrm{L}}$ and $D$ (Fig. 4C) and between $R_{\mathrm{D}}$ and $p_{\mathrm{L}, \mathrm{D}}$ (Fig. 4D), while $p_{\mathrm{L}}$ and $n$ are negatively correlated (Fig. 4C).

Table 5. Parameters of isothermal adsorption and desorption models of studied coal samples $\left(V_{\mathrm{L}}, V_{0}, V_{\mathrm{L}, \mathrm{D}}, V_{\mathrm{d}}\right.$ and $V_{\mathrm{D}}$ in $\mathrm{cm}^{3} / \mathrm{g} ; p_{\mathrm{L}}$ and $p_{\mathrm{L}}$,

\begin{tabular}{|c|c|c|c|c|c|c|c|c|c|c|}
\hline \multirow{2}{*}{ Sample } & \multicolumn{5}{|c|}{ Adsorption } & \multicolumn{5}{|c|}{ Desorption } \\
\hline & $V_{\mathrm{L}}$ & $p_{\mathrm{L}}$ & $V_{0}$ & $D$ & $n$ & $V_{\mathrm{L}, \mathrm{D}}$ & $p_{\mathrm{L}, \mathrm{D}}$ & $V_{\mathrm{d}}$ & $V_{\mathrm{D}}$ & $R_{\mathrm{D}}$ \\
\hline $2-1$ & 23.47 & 2.32 & 19.33 & 0.25 & 1.53 & 21.50 & 1.20 & 7.92 & 11.43 & 59.06 \\
\hline $2-2$ & 14.53 & 2.13 & 11.88 & 0.18 & 1.87 & 14.11 & 1.53 & 4.43 & 7.49 & 62.84 \\
\hline $2-3$ & 10.34 & 4.49 & 7.43 & 0.40 & 1.34 & 8.63 & 1.99 & 2.25 & 5.09 & 69.42 \\
\hline $2-5$ & 17.43 & 2.60 & 13.96 & 0.25 & 1.60 & 16.12 & 1.57 & 4.97 & 8.90 & 64.17 \\
\hline $2-7$ & 20.77 & 3.73 & 15.39 & 0.33 & 1.48 & 18.00 & 1.97 & 4.72 & 10.61 & 69.21 \\
\hline $2-10$ & 22.93 & 2.03 & 19.14 & 0.21 & 1.67 & 21.69 & 1.23 & 7.87 & 11.38 & 59.14 \\
\hline
\end{tabular}




\begin{tabular}{|c|c|c|c|c|c|c|c|c|c|c|}
\hline $2-12$ & 19.94 & 2.35 & 16.30 & 0.24 & 1.58 & 19.12 & 1.80 & 5.35 & 11.02 & 67.30 \\
\hline $2-13$ & 22.24 & 2.57 & 17.87 & 0.25 & 1.58 & 20.95 & 1.65 & 6.24 & 11.61 & 65.05 \\
\hline $4-3$ & 8.98 & 3.23 & 6.99 & 0.33 & 1.40 & 7.44 & 1.02 & 3.03 & 3.83 & 55.88 \\
\hline 4-4 & 12.54 & 5.75 & 8.27 & 0.44 & 1.34 & 9.38 & 1.56 & 2.91 & 5.20 & 64.17 \\
\hline $4-5$ & 14.70 & 3.27 & 11.31 & 0.31 & 1.46 & 13.12 & 1.95 & 3.47 & 7.84 & 69.35 \\
\hline 4-6 & 21.39 & 2.84 & 17.10 & 0.30 & 1.47 & 18.66 & 1.30 & 6.53 & 10.47 & 61.59 \\
\hline 4-7 & 18.12 & 2.49 & 14.58 & 0.24 & 1.65 & 17.02 & 1.61 & 5.16 & 9.35 & 64.45 \\
\hline 4-9 & 14.85 & 3.84 & 10.95 & 0.34 & 1.47 & 12.43 & 1.52 & 3.92 & 6.88 & 63.72 \\
\hline $4-11$ & 17.03 & 5.18 & 11.85 & 0.45 & 1.27 & 12.74 & 1.17 & 4.77 & 6.95 & 59.29 \\
\hline $9-1$ & 22.38 & 2.38 & 18.15 & 0.23 & 1.63 & 20.97 & 1.55 & 6.52 & 11.80 & 64.39 \\
\hline $9-2$ & 20.19 & 3.02 & 15.60 & 0.27 & 1.61 & 18.44 & 1.62 & 5.56 & 10.16 & 64.61 \\
\hline $9-3$ & 12.64 & 2.31 & 10.35 & 0.23 & 1.62 & 11.74 & 1.48 & 3.77 & 6.64 & 63.78 \\
\hline $9-4$ & 19.31 & 3.20 & 14.75 & 0.28 & 1.56 & 17.39 & 1.88 & 4.72 & 10.11 & 68.19 \\
\hline $9-5$ & 22.70 & 3.26 & 17.29 & 0.29 & 1.56 & 20.42 & 1.84 & 5.63 & 11.57 & 67.28 \\
\hline $9-7$ & 18.65 & 3.76 & 13.94 & 0.35 & 1.43 & 15.11 & 1.05 & 6.04 & 7.66 & 55.87 \\
\hline $9-8$ & 25.17 & 2.45 & 20.66 & 0.22 & 1.63 & 23.19 & 1.11 & 8.97 & 11.89 & 57.00 \\
\hline $9-9$ & 24.25 & 2.03 & 20.32 & 0.21 & 1.71 & 22.80 & 1.35 & 7.79 & 12.49 & 61.61 \\
\hline $9-10$ & 24.39 & 2.58 & 19.38 & 0.22 & 1.74 & 21.41 & 1.25 & 7.69 & 11.70 & 60.36 \\
\hline
\end{tabular}

$V_{\mathrm{L}}$, Langmuir volume; $p_{\mathrm{L}}$, Langmuir pressure; $V_{0}$, volume of micropore; $D$, constant relevant to adsorption heat; $n$, constant relevant to pore distribution; $V_{\mathrm{L}, \mathrm{D}}$, Langmuir volume in desorption; $p_{\mathrm{L}, \mathrm{D}}$, Langmuir pressure in desorption; $V_{\mathrm{d}}$, depletion gas content; $V_{\mathrm{D}}$, desorbed gas content; $R_{\mathrm{D}}$, desorption rate.
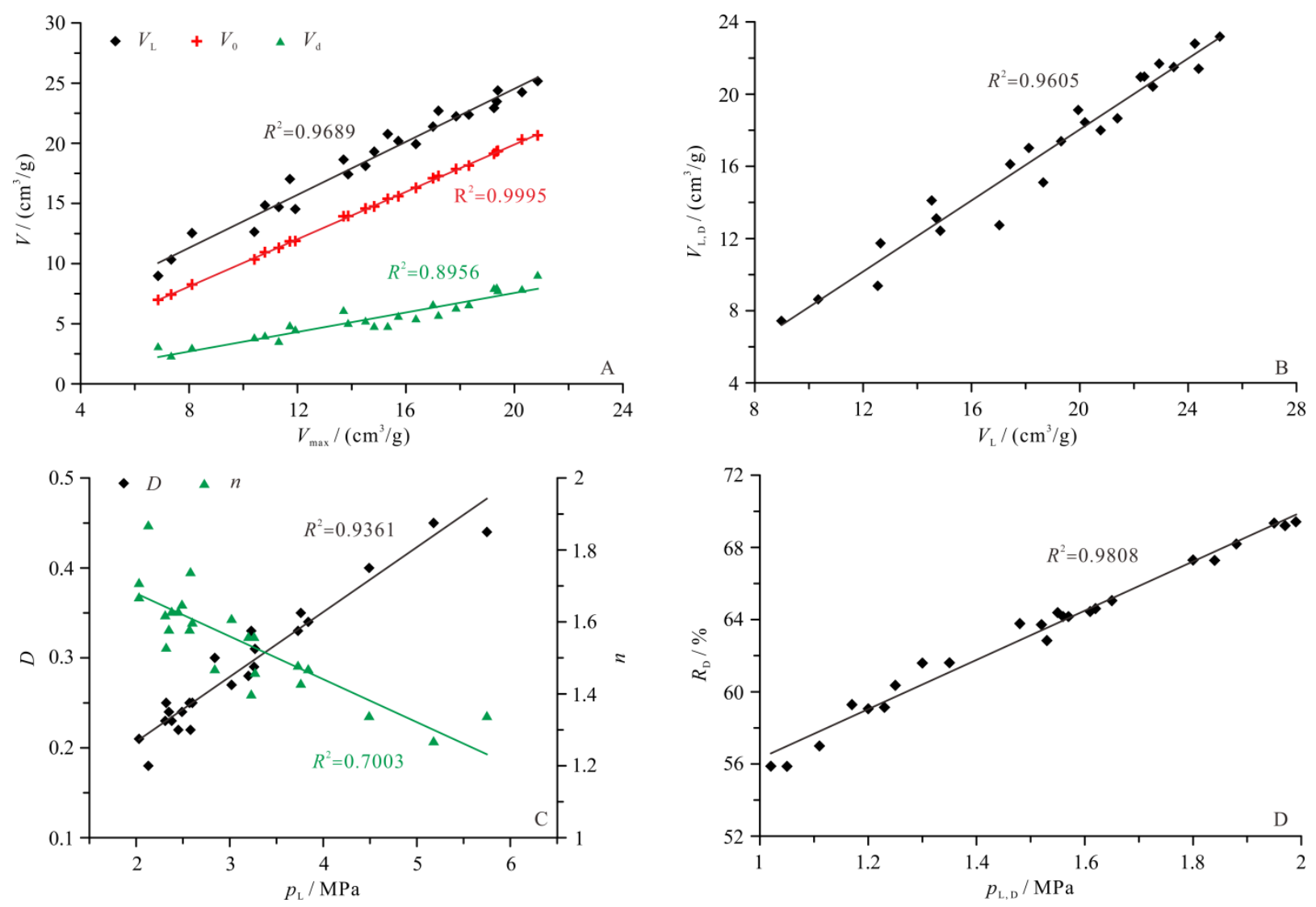

Fig. 4. Relationship between the isothermal adsorption and desorption parameters of studied coal samples.

\subsection{Proximate, micropore and sulfur analyses}

A series of auxiliary analyses were conducted to measure the moisture content, ash yield, volatile compounds, fixed carbon, pore volume and specific surface area and sulfur of the samples (Table 1). As the micropore plays a 
decisive role in gas adsorption in coal (Fu et al., 2007), pore with diameter less than $10 \mu \mathrm{m}$, which was classified as the micropore by ХОДОТ (1966), was particularly taken into consideration in this study (Table 1). Ash yield obtained from proximate analysis correlates inversely with fixed carbon, micropore volume $\left(V_{\mathrm{m}}\right)$ and specific surface area $\left(S_{\mathrm{m}}\right)$ at determination coefficients $\left(R^{2}\right)$ all higher than 0.92 (Fig. 5A and Fig. 5B). Likewise, the coefficient between ash yield and organic sulfur is 0.73 (Fig. $5 \mathrm{~A})$. Micropore volume $\left(V_{\mathrm{m}}\right)$ correlates strongly with specific surface area $\left(S_{\mathrm{m}}\right)$ at a determination coefficient of nearly 1.00 (Fig. 5C). Simultaneously, these two pore parameters positively correlate with fixed carbon and organic sulfur at determination coefficients higher than 0.69 (Fig. 5C and Fig. 5D).
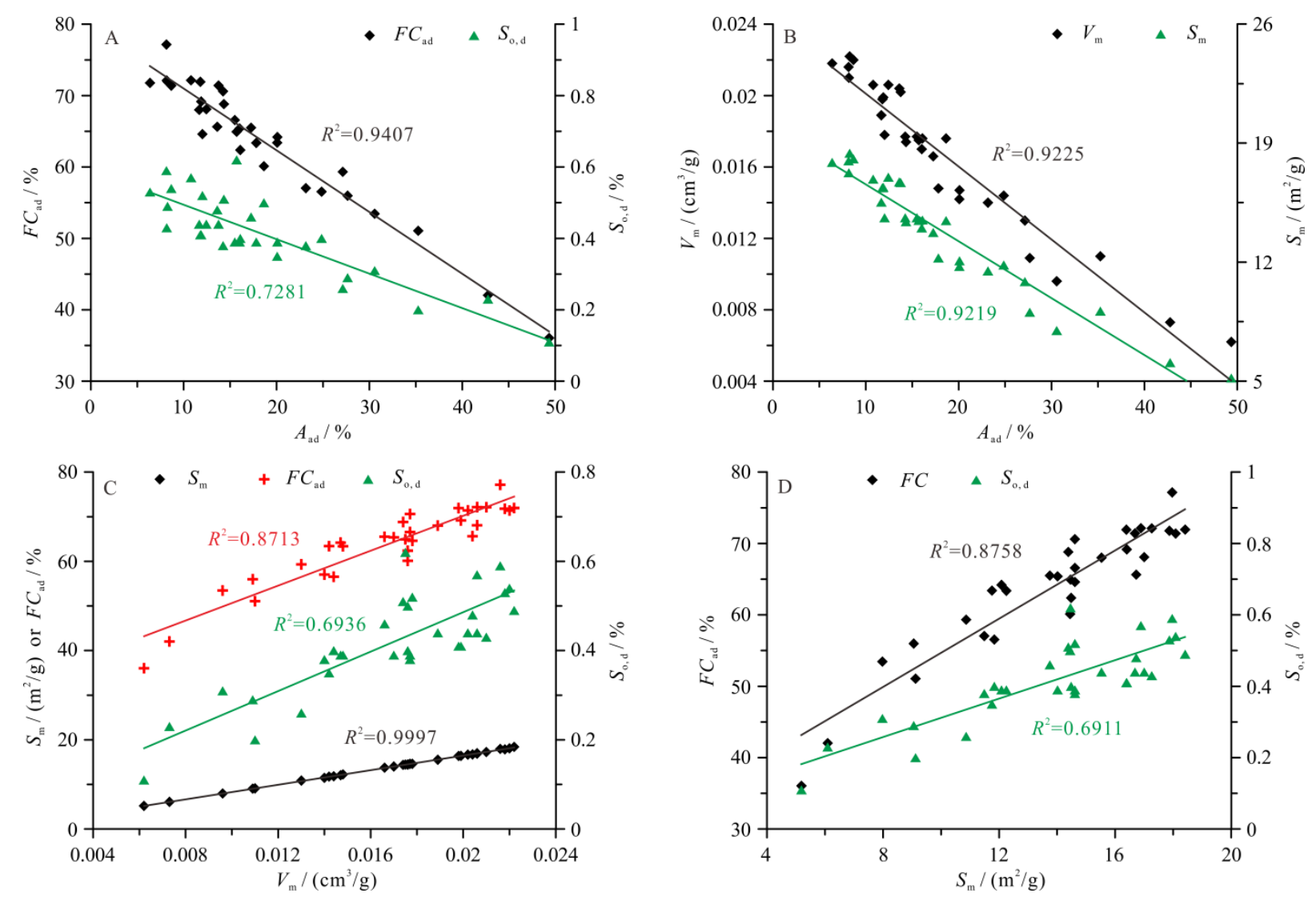

Fig. 5. Relationship between the coal quality and pore parameters of studied coal samples.

\section{Discussion}

\subsection{Types of coal facies}

\subsubsection{Terminology and methodology of paleomire}

Peat-forming environments are commonly described using several terms, such as swamp, marsh, mire, moor and bog. Moore (1987) proposed the term 'mire' as the most general and defined major categories of peat-forming systems based largely on hydrology, especially the source of water and ion composition and concentration. In general, rheotrophic mires are recharged both from groundwater and rainfall. Ombrotrophic mires, in contrast, are solely rain-fed. Moore (1987) adopted the term bog to describe the ombrotrophic type of mire while dividing rheotrophic mires into fens and swamps on the basis of flooding range with respect to the mire surface. Marshes are another type of rheotrophic mire in which herbaceous plants prevail and peat accumulation is minimal. In this paper, the terms 'mire' and 'paleomire' are used as collective descriptions of peat-forming environments in the Xiqu Drainage Area. Specific coal facies are denominated in the light of petrographic indices used as indicators of plant material and depositional conditions to define particular paleopeat-forming environments. 
Numerous schemes have been proposed in the past to enumerate the depositional environments of coal based on the quantitative relationships among the tissue and non-tissue derived constituents. Diessel (1986) established a correlation between the coal facies indicators and corresponding environments by both the Gelification Index (GI) and Tissue Preservation Index (TPI) for Permian coals in the Sydney Basin. This scheme was then adopted widely and prevailed in facies studies of coals from different areas and ages (Kalkreuth and Leckie, 1989; Strehlau, 1990; Kalkreuth et al., 1991; Lamberson et al., 1991; Obaje et al., 1994; Kalkreuth et al., 2000; Dai et al., 2007). Modifications have also been made to apply these two indices in low rank coal (Singh and Singh P.K., 1996; Sakorafa and Michailidis, 1997; Gmur and Kwiecińska, 2002; Bechtel et al., 2003; Amijaya and Littke, 2005).

The TPI is a measure of the relationship between the plant tissue and groundmass components in coal and the degree of humification, emphasizing the degree of plant cell tissue preservation versus destruction. Meanwhile, the TPI represents the effects of the input of woody material and its preservation prior to final deposition. Increased TPI values are thought to indicate a dominance of arborescent plants in the primary paleomire. The GI is the ratio of gelified and fusinitized macerals and is considered to reflect the height of the water table or the continuity in moisture availability during peat accumulation. Wet conditions of peat formation are distinguished by high GI values. The GI is also regarded as an oxidation index in the mire; a decrease in GI values implies an increase in oxidation. In this study, the TPI and GI were calculated by the formulae (modified from Diessel, 1986) given below:

TPI=(Telinite+Telocollinite+Fusinite+Semifusinite)/(Desmocollinite+Vitrodetrinite+Gelocollinite+Corpocollinite + Inertodetrinite+Macrinite)

GI=(Vitrinite+Macrinite)/(Fusinite+Semifusinite+Inertodetrinite)

Another prevalent method for evaluating coal's depositional environment was proposed by Calder et al. (1991) for Westphalian coal of Nova Scotia. A paleomire environment diagram based on a Groundwater Index (GWI) and a Vegetation Index (VI) were suggested. The GWI evaluates the intensity of rheotrophic conditions as a ratio of strongly gelified vitrinite macerals and mineral matter contents over the weakly gelified vitrinite macerals. High GWI values indicate a relatively high water table in the precursor mires and vice versa. The VI is the ratio of the macerals with remnant botanical cell structure to the matrix, detrital and granular macerals such as desmocollinite, vitrodetrinite, inertodetrinite, sporinite, cutinite and liptodetrinite. In other words, the VI represents the vegetation type by contrasting the macerals of forest affinity with those of herbaceous and marginal aquatic affinity. High VI values are indicative of forested mires, whereas low VI values suggest a deposition in a limnic environment and mainly herbaceous source material. These two measures in this study were calculated following the formulae (modified from Calder et al., 1991) below:

GWI=(Vitrodetrinite+Gelocollinite+Corpocollinite+ClayMineral+Quartz)/(Telinite+Telocollinite+Desmocollinite )

$\mathrm{VI}=($ Telinite + Telocollinite + Corpocollinite + Fusinite + Semifusinite + Resinite + Suberinite $) /($ Desmocollinite + Vitrode trinite+Inertodetrinite+Liptodetrinite+Sporinite+Cutinite)

The coal-bearing strata in the Gujiao were typically deposited in a delta plain-littoral transitional area and coal seams No. 2, No. 4 and No. 9 were principally formed under the setting of a delta. In the facies analysis of the coal seams studied, the methods based on maceral composition of coals established by Diessel (1986) and Calder et al. (1991) were applied. Facies diagrams of both the GI-TPI and GWI-VI show applicability to a certain extent in these coals. 


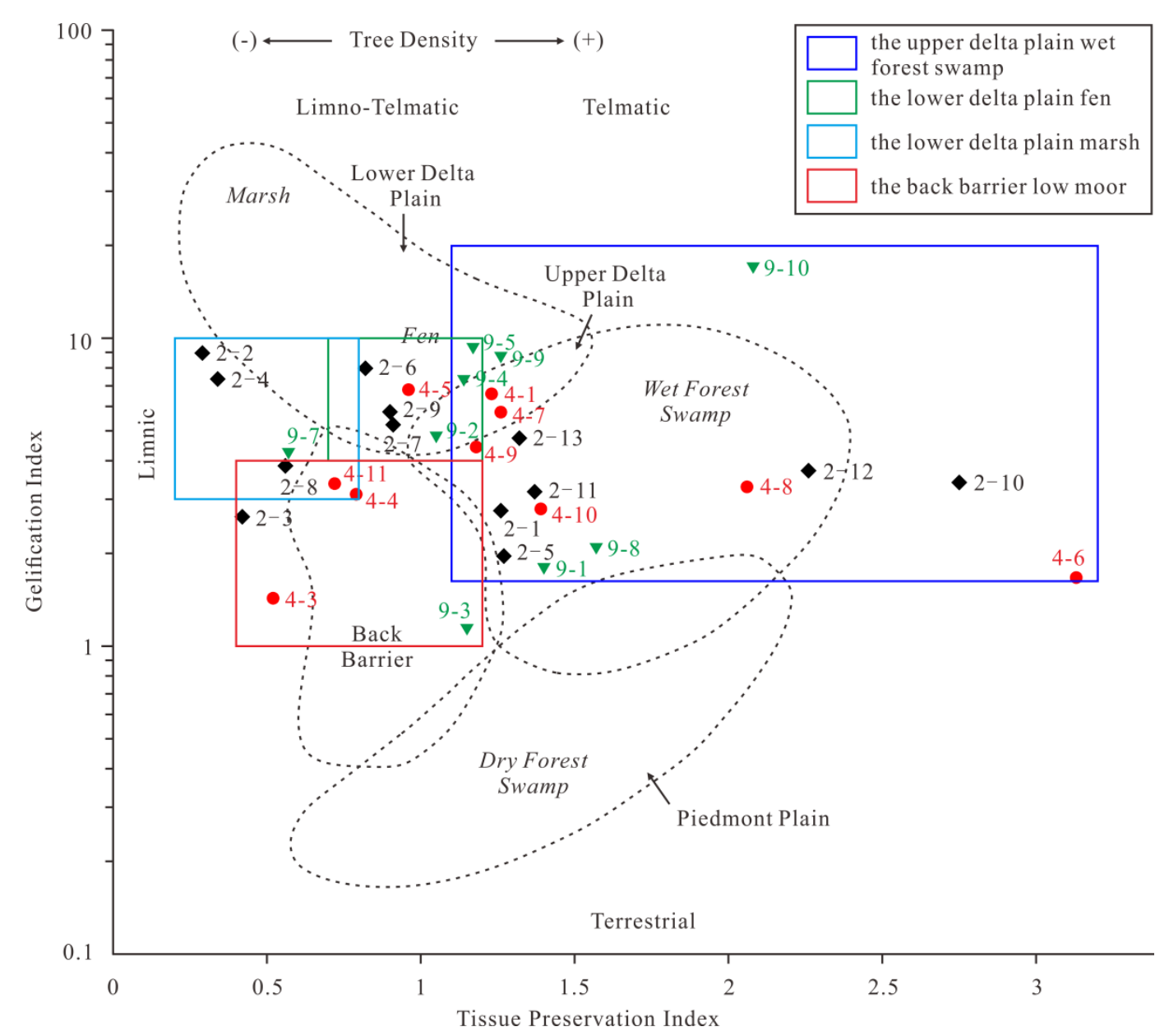

Fig. 6. GI-TPI diagram for coal facies types of studied coal samples (base map modified from Diessel, 1986).

\subsubsection{Characteristics of coal facies}

Based on the GI-TPI diagram, the coal samples examined in this study were classified into four types of coal facies (Fig. 6) and similar divisions were subsequently found in the GWI-VI diagram, even though all these types were in the ombrotrophic section (Fig. 7). These four types of coal facies are denominated as the upper delta plain wet forest swamp (I), the lower delta plain fen (II), the lower delta plain marsh (III) and the back barrier low moor (IV).

The upper delta plain wet forest swamp is the mire occupying an area of phytogenic sedimentation on the delta furthest to the sea with dominant arborescent, in fact, huge perennial plants, where both telinite and telocollinite are richer than the other three types. The coals were formed under wet condition of peat formation, and botanical structures were weakly destructed. The relatively wide ranges of the TPI and VI imply a certain variation in the plant species composition, although arborescent plants were always the prime species.

The lower delta plain fen occurs as an intermediate stage between herbaceous and forest swamp (Diessel, 1992), and a mixture of herbaceous arborescent assembly of plants supplied the peat formation under wet condition. The lower delta plain marsh is dominated by herbaceous plants growing in the vicinity of seawater. Arborescent plants were rare in this area and botanical tissues were strongly decomposed. The macerals are predominantly composed of desmocollinite of 41.6 68.5 vol\%.

The back barrier low moor is frequently influenced by seawater. The paleomire here was salinized to some extent and botanical tissues were decomposed intensely. The detrital macerals, clay mineral and pyrite in this coal facies were the richest among the four types.

Divergence and regularity among these four types of coal facies are obvious. The pyritic sulfur, sulfate sulfur, moisture content and GI are variable among different coal facies with no consistent trend in the studied coal seams. 
By contrast, the ash yield, volatile compounds, fixed carbon, organic sulfur, total sulfur, micropore volume and specific surface area, TPI, VI and GWI vary regularly among the coal facies. In general, both the ash yield and GWI tend to increase from coal facies I to IV in each coal seam, whereas the volatile compounds, fixed carbon, organic sulfur, total sulfur, micropore volume and specific surface area, TPI and VI decrease (Fig. 8).

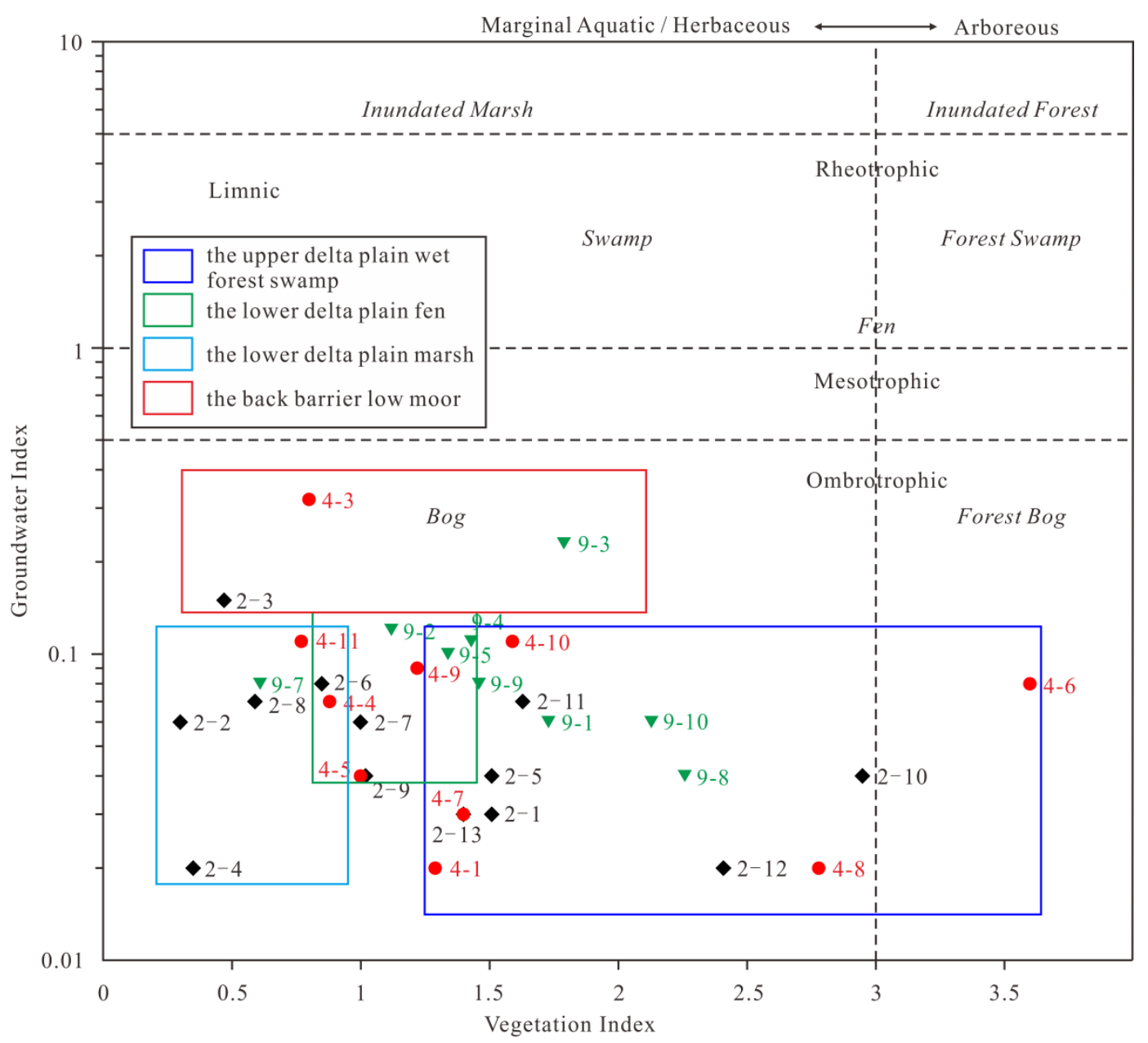

Fig. 7. GWI-VI diagram for coal facies types of studied coal samples (base map modified from Calder et al., 1991).

The paleomire environments are responsible for these variations among the coal facies. In the upper delta plain wet forest swamp, huge perennial plants prevailed in humid condition, providing abundant precursor material for coal. Meanwhile, the rapid burial of paleopeat ensured the preservation of the botanical cell structure of arborescent plants. The peat accumulated in peaceful mire water with distributary channels and crevasses seldom affected. This moist and reductive condition without frequent disturbance contributed to the preservation of organic sulfur and less extraneous mineral matter in the peat. Therefore, the ash yield and GWI in the upper delta plain wet forest swamp are low, while the volatile compounds, fixed carbon, organic sulfur, total sulfur, micropore volume and specific surface area, TPI and VI are high (Fig. 8). 


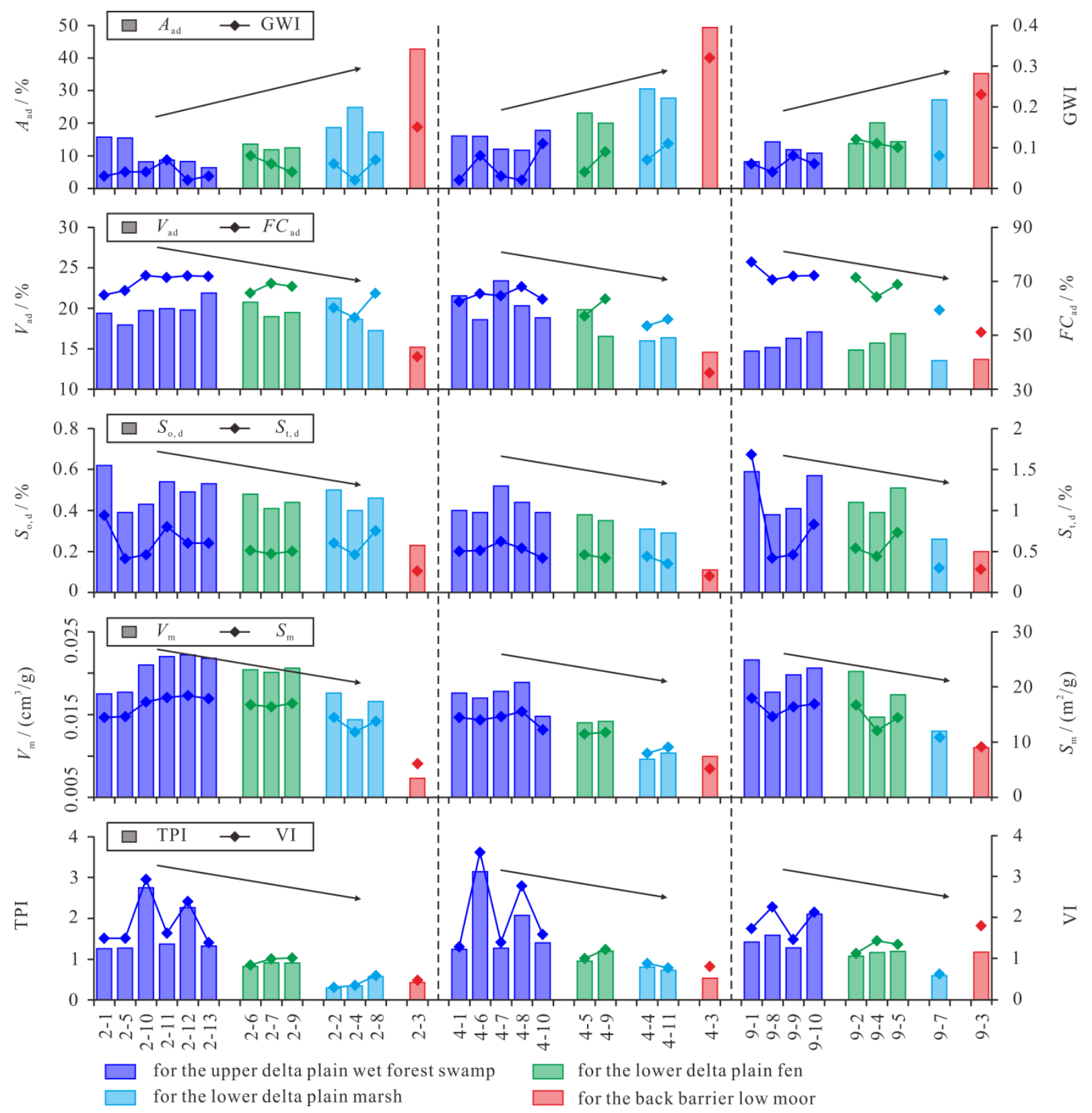

Fig. 8. Regularization parameters of coal facies of studied coal seams.

The lower delta plain fen occurs in a lower transitional area that is closer to the sea. Both herbaceous and arborescent plants supplied the peat formation under wet condition (Diessel, 1992). The botanical tissues of the herbaceous plants are more easily decomposed and made less contribution to the micropore. Occasional crevasses and overflows increased the extraneous mineral matter in the paleomire. Reductive condition for organic sulfur preservation was also affected by the intrusion of extraneous flows.

Herbaceous plants prevailed in the lower delta plain marsh and botanical tissues were strongly decomposed. More frequent extraneous flows from the distributary channels and sea brought more mineral matter to the paleomire, leading to higher ash yield and lower organic sulfur remaining (Fig. 8). In the back barrier low moor, high contents of detrital macerals, clay mineral and pyrite are attributed to marginal aquatic/herbaceous plants and salinized and active mire water.

\subsection{Paleomire evolutions}

Peat accumulation is a long process. Paleopeat-forming environments were changing with prime matter, water 
and ions supplements, acid-base properties and redox conditions (Flores, 1993; Hacquebard, 1993; Kalkreuth et al., 1993), leading to paleomire evolutions. In general, when the hydrodynamic condition is weaker, fewer terrigenous substances are brought into the mire, and the ash yield of the coal is lower. Meanwhile, peaceful mire water is always related to a relatively reductive condition that is advantageous to the gelification and decomposition of plant debris. Conversely, reactive oxygen and allochthonous deposition increase with increasing water activity, resulting in the increase of inertinite maceral group, granulated macerals (such as corpocollinite) and resinite, schistic and detrital macerals, which can reduce the organic sulfur in peat or coal. Analyses results in this study show that the organic sulfur negatively correlates with the ash yield (Fig. 8 and Fig. 9). Positive correlation is also established between the GWI and ash yield, while the TPI and ash yield are poorly correlated (Fig. 9). Therefore, the ash yield, organic sulfur and GWI are effective indicators of paleomire water conditions and the TPI is an auxiliary one.

The facies of the three coal seams studied are predominantly composed of the upper delta plain wet forest swamp (Fig. 6, Fig. 7 and Fig. 9). In addition, almost all the coal seams began and ended with this type of facies except for coal seam No. 4, which began with the lower delta plain marsh. The paleomires increased in proximity to the sea in the sequence of coal facies I to IV. Paleomire evolution in this sequence represents the process of transgression, while the inverse sequence represents regression. The maceral composition, ash yield, organic sulfur, TPI, VI and GWI all show some regularities in regard to this facies sequence (Fig. 8). According to this understanding, two, three and two evolutionary stages for coal seams No. 2, No. 4 and No. 9, respectively, can be discriminated.

For coal seam No. 2, stage 2-I began with the upper delta plain wet forest swamp of sample 2-13 (Fig. 9) overlying the coal seam floor of dark carbonaceous mudstone (Fig. 2). The paleomire changed little until the sea level rose slowly and the lower delta plain fen of sample 2-9 took advantage. Likewise, the lower delta plain marsh of sample 2-8 soon replaced it. Then, the sea level dropped, and the lower delta plain fen of sample 2-7 prevailed again. This stage finally ended with the upper delta plain wet forest swamp of sample 2-5. Stage 2-II started right after the end of stage 2-I. The sea level rose so quickly that the paleomire was replaced by the lower delta marsh directly and soon by the back barrier low moor. During the second half of this stage, which was just the symmetrical version of the first half of this stage, the lower delta plain marsh and the upper delta plain wet forest swamp quickly prevailed in the paleomires successively. The peat-forming environments then finished and dark mudstone deposited as the coal seam roof (Fig. 2).

Paleomires in coal seam No. 4 developed in three stages (Fig. 9). Stage 4-I was not a complete coal facies cycle and was composed merely of the lower delta plain marsh of sample 4-11. Both stage 4-II and stage 4-III were the complete cycles with the processes of transgression and regression. Stage 4-II proceeded rapidly, and in it quick transformations occurred between the upper delta plain wet forest swamp and the lower delta plain fen. During the comparatively long and complicated stage 4-III, the steady sea level promoted the occurrence of the upper delta plain wet forest swamp of samples 4-7 and 4-6. Along with slow rising of the sea level, the lower delta plain fen, the lower delta plain marsh and the back barrier low moor successively thrived. Gray mudstone parting of sample 4-2 deposited during the highest sea level. In the regression process, the sea level dropped so quickly that the upper delta plain wet forest swamp prevailed subsequently. The paleomires terminated by silty mudstone deposition as the coal seam roof (Fig. 2).

Paleopeat of coal seam No. 9 formed in two complete cycles with the sea level rise and drop (Fig. 9). Stage 9-I started with the steady upper delta plain wet forest swamp overlying the mudstone deposition (Fig. 2). Fast sea level rise later led to the thriving of the lower delta plain marsh of sample 9-7 and then the mudstone parting of sample 9-6. Afterwards, a quick drop of the sea level led to the development in the lower delta plain fen of sample 9-5. Paleomires changed quickly in stage 9-II. The lower delta plain fen of sample 9-4 was directly replaced by the back barrier low moor of sample 9-3. Likewise, during the regression, the lower delta plain fen and the upper delta plain wet forest swamp became dominant successively. Finally, carbonaceous mudstone and mudstone deposited as the coal seam roof (Fig. 2). 


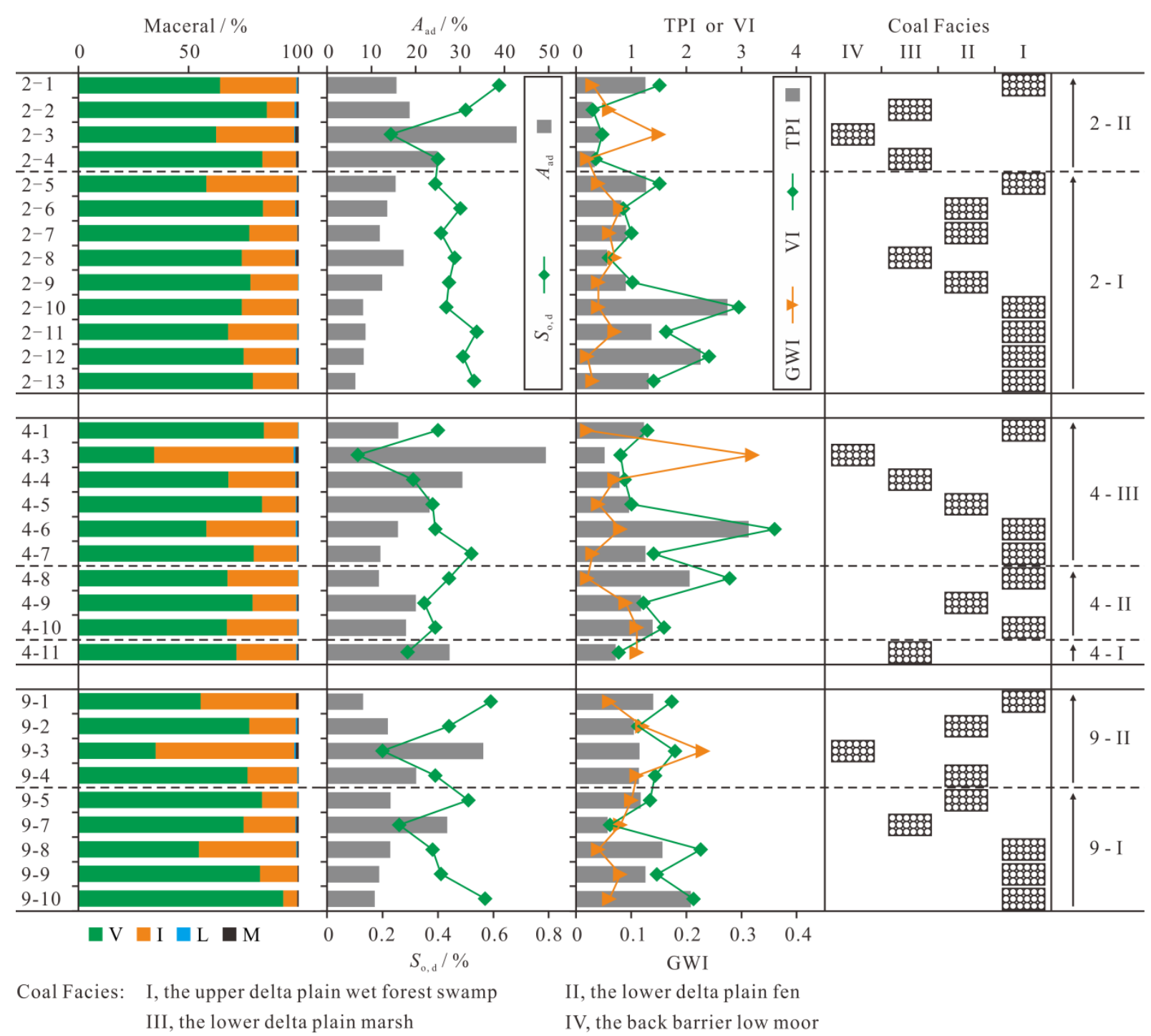

Fig. 9. Columnar sequences of coal facies and corresponding parameters of studied coal seams.

\subsection{Adsorption and desorption divergences among coal facies}

As adsorption and desorption behaviors of coal were influenced by the coalification (Yee et al., 1993), divergences of adsorption and desorption among coal facies in the three coal seams will be discussed separately due to different $R_{\mathrm{o}, \max }$ among them (Table 1). The parameters of the coal samples from the Langmuir equation and D-A equation are listed in Table 6 and Fig. 10.

Effective parameters of the Langmuir equation and D-A equation were taken as the representations for the adsorption models of monolayer and adsorption potential theory, respectively. Both $V_{\mathrm{L}}$ and $V_{0}$ represent the ultimate adsorption capacity of the coals in these equations. As shown in Table 6 and Fig. 10, $V_{\mathrm{L}}$ and $V_{0}$ decrease from coal facies I to IV. As a comprehensive indicator of the adsorption rate and desorption rate, $p_{\mathrm{L}}$, which is actually a function of the temperature and adsorption heat (Fu et al., 2007), changes in almost the same trend as the adsorption heat index $D$, while changing inversely with the pore distribution index $n$ (Fig. 4C; Table 6). Fluctuations of these three parameters exist among the coal facies with no consistent trends in the studied coal seams (Table 6 and Fig. 10).

Characteristics of desorption have also been described by the Langmuir equation. The desorbed gas content $\left(V_{\mathrm{D}}\right)$ is in agreement with the $V_{\mathrm{L}}$ and $V_{0}$ in the adsorption process (Table 6 and Fig. 10), indicating larger adsorption amounts from the respective coals correlate with stronger desorption capacities. The $p_{\mathrm{L}, \mathrm{D}}$ and $R_{\mathrm{D}}$ are well correlated (Fig. 4D) but their variation trends among the coal facies are not uniform in these three coal seams (Fig. 10). 


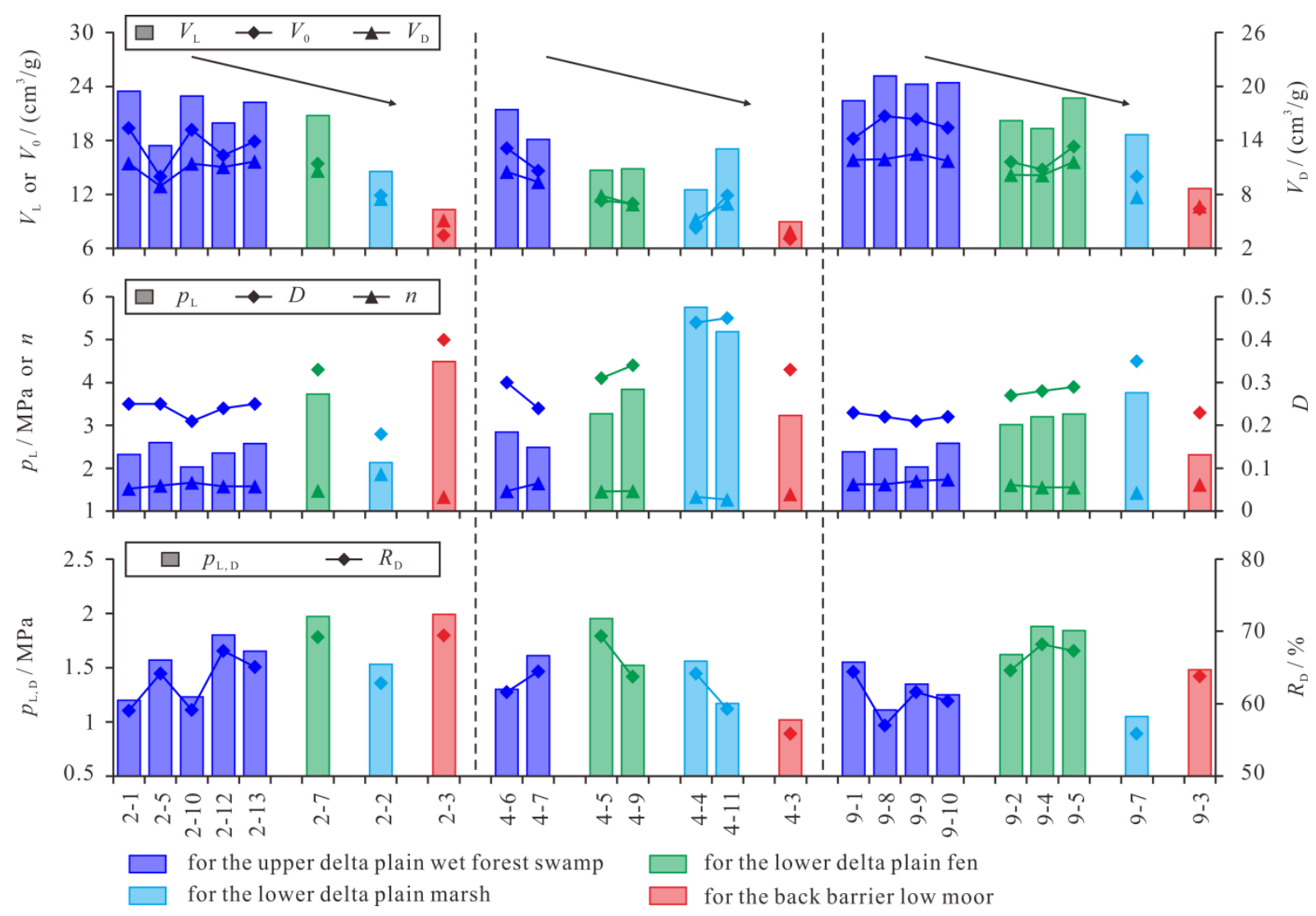

Fig. 10. Regularization isothermal adsorption-desorption parameters of coal facies of studied coal seams.

Adsorption and desorption divergences among different coal facies result from different paleomire environments, especially the variation of vegetation types and mire water condition. The arborescent plants decrease from coal facies I to IV along with the increase of hydrodynamic intensity, leading to corresponding changes in botanical tissue preservation, medium oxidizability, mineral content and ultimately, adsorption and desorption behaviors (Fig. 11). Therefore, contributing to the highest adsorption and desorption capacities are the rapid accumulation and burial of paleopeat with little mineral matter due to abundant precursor material and peaceful and reductive mire water in the upper delta plain wet forest swamp. In the lower delta plain fen and marsh, the prevailing herbaceous plants were more easily decomposed during coalification and the paleopeat also accumulated and buried slowly (Moore, 1987). The more intense hydrodynamic condition of these facies also result in a more oxidized medium and richer mineral matter. Thus, adsorption and desorption capacities of coal from the lower delta plain fen and marsh are relatively lower. In the back barrier low moor, the dominant marginal aquatic/herbaceous plants were intensely decomposed and the paleomire water was active. As ash yield from coal is counterproductive to the gas adsorption in coal (Ozawa et al., 1976; Laxminarayana and Crosdale, 1999), the lowest micropore volume and specific surface area and highest ash yield of the coal from the back barrier low moor (Fig. 8) are not conducive to gas adsorption in coal.

The adsorption heat-related parameters $p_{\mathrm{L}}$ and $D$ and the pore distribution index $n$ lack consistent variation trends among the coal facies in the studied coal seams (Table 6 and Fig. 10) owing to their intricate relations to the plant species, ash yield, redox conditions, etc. 


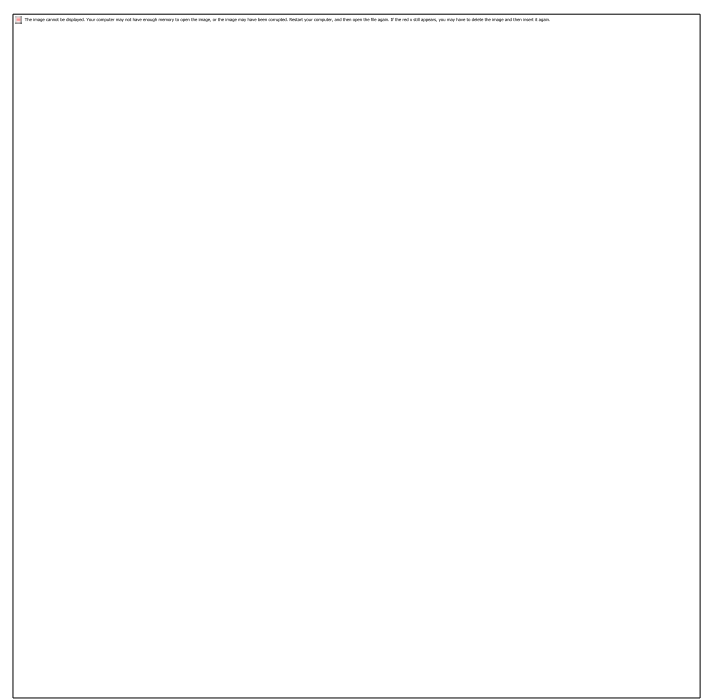

Fig. 11. Simple relationship between coal facies and adsorption-desorption behaviors. Note: I, the upper delta plain wet forest swamp; II, the lower delta plain fen; III, the lower delta plain marsh; IV, the back barrier low moor; APA, arborescent plants abundance; TPD, tissue preservation degree; HI, hydrodynamic intensity; MO, medium oxidizability; MC, mineral content; AC, adsorption capacity; DC, desorption capacity; and DP, drainage potential.

\subsection{Application of coal facies to CBM industry}

On the above basis, the adsorption and desorption behaviors of the delta plain-littoral transitional coal are obviously influenced by coal facies. In the same coal seam, gas contents of coals reduce gradually with the increasing sea water influence to the paleomires. Coal layers from the upper delta plain wet forest swamp, with the highest gas content and strongest desorption capacity, have the best CBM drainage potential (Fig. 11). Coal layers from both the lower delta plain fen and marsh, with the higher gas content and stronger desorption capacity, also have better CBM drainage potential. However, coal layers from the back barrier low moor might lose the drainage value due to the low gas content.

Unfortunately, the experiments in this study were only measured on an air-dry basis. Further work can be conducted on moisture-equilibrium condition to obtain more applicable results. 
Table 6. Statistics of the isothermal adsorption-desorption parameters of the coal facies of studied coal seams $\left(V_{\mathrm{L}}, V_{0}\right.$ and $V_{\mathrm{D}}$ in $\mathrm{cm}^{3} / \mathrm{g} ; p_{\mathrm{L}}$ and $p_{\mathrm{L}, \mathrm{D}}$ in $\mathrm{MPa}$; and $R_{\mathrm{D}}$ in $\left.\%\right)$.

\begin{tabular}{|c|c|c|c|c|c|c|c|c|c|c|c|c|c|c|c|c|c|}
\hline \multirow{12}{*}{ No. 2} & \multirow{2}{*}{ Coal facies } & \multicolumn{4}{|c|}{$V_{\mathrm{L}}$} & \multicolumn{4}{|c|}{$V_{0}$} & \multicolumn{4}{|c|}{$V_{\mathrm{D}}$} & \multicolumn{4}{|c|}{$p_{\mathrm{L}}$} \\
\hline & & Min & Max & Mean & StDev & Min & Max & Mean & StDev & Min & Max & Mean & StDev & Min & Max & Mean & StDev \\
\hline & I & 17.43 & 23.47 & 21.20 & 2.24 & 13.96 & 19.33 & 17.32 & 2.00 & 8.90 & 11.61 & 10.87 & 1.00 & 2.03 & 2.60 & 2.37 & 0.21 \\
\hline & II & 20.77 & 20.77 & 20.77 & 0 & 15.39 & 15.39 & 15.39 & 0 & 10.61 & 10.61 & 10.61 & 0 & 3.73 & 3.73 & 3.73 & 0 \\
\hline & III & 14.53 & 14.53 & 14.53 & 0 & 11.88 & 11.88 & 11.88 & 0 & 7.49 & 7.49 & 7.49 & 0 & 2.13 & 2.13 & 2.13 & 0 \\
\hline & IV & 10.34 & 10.34 & 10.34 & 0 & 7.43 & 7.43 & 7.43 & 0 & 5.09 & 5.09 & 5.09 & 0 & 4.49 & 4.49 & 4.49 & 0 \\
\hline & \multirow{2}{*}{ Coal facies } & \multicolumn{4}{|c|}{$D$} & \multicolumn{4}{|c|}{$n$} & \multicolumn{4}{|c|}{$p_{\mathrm{L}, \mathrm{D}}$} & \multicolumn{4}{|c|}{$R_{\mathrm{D}}$} \\
\hline & & Min & $\operatorname{Max}$ & Mean & StDev & Min & Max & Mean & StDev & Min & $\operatorname{Max}$ & Mean & StDev & Min & Max & Mean & StDev \\
\hline & I & 0.21 & 0.25 & 0.24 & 0.02 & 1.53 & 1.67 & 1.59 & 0.05 & 1.20 & 1.80 & 1.49 & 0.24 & 59.06 & 67.30 & 62.94 & 3.30 \\
\hline & II & 0.33 & 0.33 & 0.33 & 0 & 1.48 & 1.48 & 1.48 & 0 & 1.97 & 1.97 & 1.97 & 0 & 69.21 & 69.21 & 69.21 & 0 \\
\hline & III & 0.18 & 0.18 & 0.18 & 0 & 1.87 & 1.87 & 1.87 & 0 & 1.53 & 1.53 & 1.53 & 0 & 62.84 & 62.84 & 62.84 & 0 \\
\hline & IV & 0.40 & 0.40 & 0.40 & 0 & 1.34 & 1.34 & 1.34 & 0 & 1.99 & 1.99 & 1.99 & 0 & 69.42 & 69.42 & 69.42 & 0 \\
\hline \multirow{12}{*}{ No. 4} & \multirow{2}{*}{ Coal facies } & \multicolumn{4}{|c|}{$V_{\mathrm{L}}$} & \multicolumn{4}{|c|}{$V_{0}$} & \multicolumn{4}{|c|}{$V_{\mathrm{D}}$} & \multicolumn{4}{|c|}{$p_{\mathrm{L}}$} \\
\hline & & Min & Max & Mean & StDev & Min & Max & Mean & StDev & Min & Max & Mean & StDev & Min & Max & Mean & StDev \\
\hline & I & 18.12 & 21.39 & 19.76 & 1.64 & 14.58 & 17.10 & 15.84 & 1.26 & 9.35 & 10.47 & 9.91 & 0.56 & 2.49 & 2.84 & 2.67 & 0.18 \\
\hline & II & 14.70 & 14.85 & 14.78 & 0.08 & 10.95 & 11.31 & 11.13 & 0.18 & 6.88 & 7.84 & 7.36 & 0.48 & 3.27 & 3.84 & 3.56 & 0.29 \\
\hline & III & 12.54 & 17.03 & 14.79 & 2.25 & 8.27 & 11.85 & 10.06 & 1.79 & 5.20 & 6.95 & 6.08 & 0.88 & 5.18 & 5.75 & 5.47 & 0.29 \\
\hline & IV & 8.98 & 8.98 & 8.98 & 0 & 6.99 & 6.99 & 6.99 & 0 & 3.83 & 3.83 & 3.83 & 0 & 3.23 & 3.23 & 3.23 & 0 \\
\hline & \multirow{2}{*}{ Coal facies } & \multicolumn{4}{|c|}{$D$} & \multicolumn{4}{|c|}{$n$} & \multicolumn{4}{|c|}{$p_{\mathrm{L}, \mathrm{D}}$} & \multicolumn{4}{|c|}{$R_{\mathrm{D}}$} \\
\hline & & Min & Max & Mean & StDev & Min & Max & Mean & StDev & Min & Max & Mean & StDev & Min & Max & Mean & StDev \\
\hline & I & 0.24 & 0.30 & 0.27 & 0.03 & 1.47 & 1.65 & 1.56 & 0.09 & 1.30 & 1.61 & 1.46 & 0.16 & 61.59 & 64.45 & 63.02 & 1.43 \\
\hline & II & 0.31 & 0.34 & 0.33 & 0.02 & 1.46 & 1.47 & 1.47 & 0.01 & 1.52 & 1.95 & 1.74 & 0.22 & 63.72 & 69.35 & 66.54 & 2.82 \\
\hline & III & 0.44 & 0.45 & 0.45 & 0.01 & 1.27 & 1.34 & 1.31 & 0.04 & 1.17 & 1.56 & 1.37 & 0.20 & 59.29 & 64.17 & 61.73 & 2.44 \\
\hline & IV & 0.33 & 0.33 & 0.33 & 0 & 1.4 & 1.4 & 1.4 & 0 & 1.02 & 1.02 & 1.02 & 0 & 55.88 & 55.88 & 55.88 & 0 \\
\hline \multirow{8}{*}{ No. 9} & \multirow{2}{*}{ Coal facies } & & & & & & & & & & & & & & & & \\
\hline & & Min & Max & Mean & StDev & Min & $\operatorname{Max}$ & Mean & StDev & Min & $\operatorname{Max}$ & Mean & StDev & Min & $\operatorname{Max}$ & Mean & StDev \\
\hline & I & 22.38 & 25.17 & 24.05 & 1.02 & 18.15 & 20.66 & 19.63 & 0.97 & 11.70 & 12.49 & 11.97 & 0.31 & 2.03 & 2.58 & 2.36 & 0.20 \\
\hline & II & 19.31 & 22.70 & 20.73 & 1.44 & 14.75 & 17.29 & 15.88 & 1.06 & 10.11 & 11.57 & 10.61 & 0.68 & 3.02 & 3.26 & 3.16 & 0.10 \\
\hline & III & 18.65 & 18.65 & 18.65 & 0 & 13.94 & 13.94 & 13.94 & 0 & 7.66 & 7.66 & 7.66 & 0 & 3.76 & 3.76 & 3.76 & 0 \\
\hline & IV & 12.64 & 12.64 & 12.64 & 0 & 10.35 & 10.35 & 10.35 & 0 & 6.64 & 6.64 & 6.64 & 0 & 2.31 & 2.31 & 2.31 & 0 \\
\hline & Copl forio & & & & & & & & & & & & & & & & \\
\hline & Coal racies & Min & $\operatorname{Max}$ & Mean & StDev & Min & $\operatorname{Max}$ & Mean & StDev & Min & $\operatorname{Max}$ & Mean & StDev & Min & $\operatorname{Max}$ & Mean & StDev \\
\hline
\end{tabular}




\begin{tabular}{|c|c|c|c|c|c|c|c|c|c|c|c|c|c|c|c|c|}
\hline I & 0.21 & 0.23 & 0.22 & 0.01 & 1.63 & 1.74 & 1.68 & 0.05 & 1.11 & 1.55 & 1.32 & 0.16 & 57.00 & 64.39 & 60.84 & 2.65 \\
\hline II & 0.27 & 0.29 & 0.28 & 0.01 & 1.56 & 1.61 & 1.58 & 0.02 & 1.62 & 1.88 & 1.78 & 0.11 & 64.61 & 68.19 & 66.69 & 1.52 \\
\hline III & 0.35 & 0.35 & 0.35 & 0 & 1.43 & 1.43 & 1.43 & 0 & 1.05 & 1.05 & 1.05 & 0 & 55.87 & 55.87 & 55.87 & 0 \\
\hline IV & 0.23 & 0.23 & 0.23 & 0 & 1.62 & 1.62 & 1.62 & 0 & 1.48 & 1.48 & 1.48 & 0 & 63.78 & 63.78 & 63.78 & 0 \\
\hline
\end{tabular}

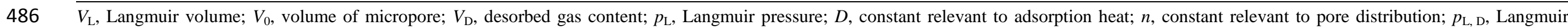

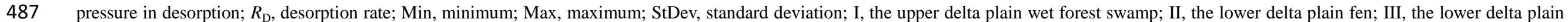
488 marsh; and IV, the back barrier low moor. 
Four types of coal facies were distinguished based on the GI-TPI and GWI-VI diagrams, including the upper delta plain wet forest swamp (I), the lower delta plain fen (II), the lower delta plain marsh (III) and the back barrier low moor (IV), in coal seams No. 2, No. 4 and No. 9 in the Xiqu Drainage Area, Gujiao CBM Block, North China. The upper delta plain wet forest swamp occurred dominantly, developing at the bottom and top of all the coal seams. Both the ash yield and GWI tend to increase from coal facies I to IV, whereas the volatile compounds, fixed carbon, organic sulfur, total sulfur, micropore volume and specific surface area, TPI and VI decreased. The vegetation types, water supplements and disturbance, acid-base properties and redox conditions of the paleomires were responsible for these divergences.

Paleomire evolution accompanied the rise and drop of the sea level, with a transgression or regression process that could be represented by the coal facies sequence. Coal seams No. 2, No. 4 and No. 9 were discriminated into two, three and two evolving stages, respectively, and these stages were characterized by complete cycles except stage 4-I from the lower delta plain marsh. Mudstone partings in the coal seams deposited during the highest sea level.

In general, with the paleomire milieu change, especially the decrease of arborescent plants and increase of mire water activity from the coal facies I to IV in each coal seam, the ultimate adsorption capacity and desorbed gas content of coals decreased. Intricate influence of the plant species, terrigenous input and redox conditions of the paleomire led to the lack of consistent evolution trends of the adsorption heat-related parameters $p_{\mathrm{L}}$ and $D$ and the pore distribution index $n$. In view of the gas content and desorption capacity of the coal layers in the same coal seam, the layers from the upper delta plain wet forest swamp may have the best potential for CBM drainage, followed by those from the lower delta plain fen and marsh, while those from the back barrier low moor may have the worst potential.

\section{Acknowledgements}

We would like to thank the Geology Section of Xishan Coal-Electricity Group Co., Ltd for collecting the channel coal samples and Dr. Y.G. Chen for the data processing instruction. This research was financially supported by the key projects of the National Natural Science Foundation of China (No. 41530314 and No. U1361207) and the National Science Foundation for Distinguished Young Scholars of China (No. 41125009).

\section{References}

Amijaya, H., Littke, R., 2005. Microfacies and depositional environment of Tertiary TanjungEnim low rank coal, South Sumatra Basin, Indonesia. International Journal of Coal Geology 61 (3-4), 197-221.

Bechtel, A., Gruber, W., Sachsenhofer, R.F., Gratzer, R., Lücke, A., Püttmann, W., 2003. Depositional environment of Late Miocene Hausruck lignite (Alpine Foreland Basin): insights from petrography, organic geochemistry, and stable carbon isotopes. International Journal of Coal Geology 53 (3), 153-180.

Calder, J.H., Gibling, M.R., Mukhopadhyay, P.K., 1991. Peat formation in a Westphalian B piedmont setting, Cumberland Basin, Nova Scotia: Implications for the maceral-based interpretation of rheotrophic and raised paleomires. Bulletin de la Société Géologique de France 162 (2), 283-298.

Dai, S.F., Ren, D.Y., Li, S.S., Zhao, L., Zhang, Y., 2007. Coal facies evolution of the main minable coal-bed in the Heidaigou Mine, Jungar Coalfield, Inner Mongolia, northern China. Science in China Series D: Earth Sciences 50 (S2), 144-152.

Daines, M.E., 1968. Apparatus for the determination of methane sorption on coal at high pressures by a weighing method. International Journal of Rock Mechanics and Mining Sciences 5 (4), 315-316.

Day, S., Sakurovs, R., Weir, S., 2008. Supercritical gas sorption on moist coals. International Journal of Coal Geology 74 (3-4), $203-214$.

Diessel, C.F.K., 1982. An appraisal of coal facies based on maceral characteristics. Australian Coal Geology, 4 (2), 474-484.

Diessel, C.F.K., 1986. The correlation between coal facies and depositional environments, in: Advances in the study of the Sydney Basin, Proceedings of 20th Symposium. The University of Newcastle, Newcastle, pp. 19-22.

Diessel, C.F.K., 1992. Coal-bearing Depositional Systems. Springer-Verlag, Berlin.

Diessel, C.F.K., 2007. Utility of coal petrology for sequence-stratigraphic analysis. International Journal of Coal Geology 70 (1-3), 3-34.

DiMichele, W.A., Phillips, T.L., 1994. Paleobotanical and paleoecological constraints on models of peat formation in the Late Carboniferous of Euramerica. Palaeogeography, Palaeoclimatology, Palaeoecology 106 (1-4), 39-90.

Flores, R.M., 1993. Geologic and geomorphic controls of coal development in some Tertiary Rocky Mountain basins, USA. International Journal of Coal Geology 23 (1-4), 43-73. 
Flores, R.M., 1998. Coalbed methane: From hazard to resource. International Journal of Coal Geology 35 (1-4), 3-26.

Fu, X.H., Qin, Y., Wei, C.T., 2007. Coaloed Gas Geology. China University of Mining and Technology Press, Xuzhou. (in Chinese)

Gmur, D., Kwiecińska, B.K., 2002. Facies analysis of coal seams from the Cracow Sandstone Series of the Upper Silesia Coal Basin, Poland. International Journal of Coal Geology 52 (1-4), 29-44.

Hacquebard, P.A., 1993. Petrology and facies studies of the Carboniferous coals at Mabou Mines and inverness in comparison with those of the Port Hood, St. Rose and Sydney coalfields of Cape Breton Island, Nova Scotia, Canada. International Journal of Coal Geology 24 (1-4), 7-46.

Hacquebard, P.A., Donaldson, J.R., 1969. Carboniferous Coal Deposition Associated with Flood-Plain and Limnic Environments in Nova Scotia. Geological Society of America Special Papers 114, 143-192.

Han, D.X., Ren, D.Y., Wang, Y.B., Jin, K.L., Mao, H.L., Qin, Y., 1996. Coal Petrology of China. China University of Mining and Technology Press, Xuzhou, pp. 218. (in Chinese with an English abstract)

ISO 11760: 2005, 2005. Classification of coals.

ISO 7404-3: 2009, 2009. Methods for the petrographic analysis of coals -- Part 3: Method of determing maceral group composition.

Joubert, J.I., Grein, C.T., Bienstock, D., 1973. Sorption of methane in moist coal. Fuel 52 (3), 181-185.

Kalkreuth, W., Leckie, D.A., 1989. Sedimentological and petrographical characteristics of Cretaceous strandplain coals: a model for coal accumulation from the North American Western Interior Seaway. International Journal of Coal Geology 12 (1-4), 381-424.

Kalkreuth, W., Marchioni, D., Utting, J., 2000. Petrology, palynology, coal facies, and depositional environments of an Upper Carboniferous coal seam, Minto Coalfield, New Brunswick, Canada. Canadian Journal of Earth Sciences 37 (9), 1209-1228.

Kalkreuth, W.D., Marchioni, D.L., Calder, J.H., Lamberson, M.N., Naylor, R.D., Paul, J., 1991. The relationship between coal petrography and depositional environments from selected coal basins in Canada. International Journal of Coal Geology 19 (1-4), 21-76.

Kalkreuth, W.D., Mclntyre, D.J., Richardson, R.J.H., 1993. The geology, petrography and palynology of Tertiary coals from the Eureka Sound Group at Strathcona Fiord and Bache Peninsula, Ellesmere Island, Arctic Canada. International Journal of Coal Geology 24 (1-4), 75-111.

Lamberson, M.N., Bustin, R.M., Kalkreuth, W., 1991. Lithotype (maceral) composition and variation as correlated with paleo-wetland environments, Gates Formation, northeastern British Columbia, Canada. International Journal of Coal Geology 18 (1-2), 87-124.

Langmuir, I., 1918. The adsorption of gases on plane surfaces of glass, mica and platinum. Journal of the American Chemical Society 40, 1361-1403.

Laxminarayana, C., Crosdale, P.J., 1999. Role of coal type and rank on methane sorption characteristics of Bowen Basin, Australia coals. International Journal of Coal Geology 40 (4), 309-325.

Marchioni, D., Kalkreuth, W., 1991. Coal facies interpretations based on lithotype and maceral variations in Lower Cretaceous (Gates Formation) coals of Western Canada. International Journal of Coal Geology 18 (1-2), 125-162.

Marchioni, D.L., 1980. Petrography and depositional environment of the liddell seam, upper hunter valley, New South Wales. International Journal of Coal Geology 1 (1), 35-61.

Michaelsen, P., Henderson, R.A., 2000. Facies relationships and cyclicity of high-latitude, Late Permian coal measures, Bowen Basin, Australia. International Journal of Coal Geology 44 (1), 19-48.

Moore, P.D., 1987. Ecological and hydrological aspects of peat formation, in: Scott A.C. (Ed), Coal and Coal-Bearing Strata: Recent Advances. Geological Society London Special Publications 32, pp. 7-15.

Nowak, G.J., Górecka-Nowak, A., 1999. Peat-forming environments of Westphalian A coal seams from the Lower Silesian Coal Basin of SW Poland based on petrographic and palynologic data. International Journal of Coal Geology 40 (4), 327-351.

Obaje, N.G., Ligouis, B., 1996. Petrographic evaluation of the depositional environments of the Cretaceous Obi/Lafia coal deposits in the Benue trough of Nigeria. Journal of African Earth Sciences 22 (2), 159-171.

Obaje, N.G., Ligouis, B., Abaa, S.I., 1994. Petrographic composition and depositional environments of Cretaceous coals and coal measures in the Middle Benue Trough of Nigeria. International Journal of Coal Geology 26 (3-4), 233-260.

Ozawa, S., Kusumi, S., Ogino, Y., 1976. Physical adsorption of gases at high pressure. IV. An improvement of the Dubinin-Astakhov adsorption equation. Journal of Colloid and Interface Science 56 (1), 83-91.

Sakorafa, V., Michailidis, K., 1997. The geology and coal petrology of a Pleistocene lignite profile at Horemi mine, Megalopolis basin, Peloponnese (southern Greece). International Journal of Coal Geology 33 (1), 73-91.

Singh, M.P., Singh, P.K., 1996. Petrographic characterization and evolution of the Permian coal deposits of the Rajmahal basin, Bihar,India. International Journal of Coal Geology 29 (1-3), 93-118.

Strehlau K., 1990. Facies and genesis of Carboniferous coal seams of Northwest Germany. International Journal of Coal Geology 15 (4), 245-292.

Taylor, G.H., Teichmüller, M., Davis, A., Diessel, C.F.K., Littke, R., Robert, P., 1998. Organic Petrology. Gebrüder Borntraeger, Berlin.

Teichmüller, M., 1989. The genesis of coal from the viewpoint of coal petrology. International Journal of Coal Geology 12 (1-4), 1-87.

Wang, C.Y., Hao, S.X., Sun, W.J., Chu, W., 2012. Fractal dimension of coal particles and their CH4 adsorption. International Journal of Mining Science and Technology 22 (6), 855-858.

Wang, G., Qin, Y., Xie, Y.W., Shen, J., Han, B.B., Huang, B., Zhao, L., 2015. The division and geologic controlling factors of a vertical superimposed coalbed methane system in the northern Gujiao Blocks, China. Journal of Natural Gas Science and Engineering 24, 379-389.

Xu, H., Tang, D.Z., Liu, D.M., Tang, S.H., Yang, F., Chen, X.Z., He, W., Deng, C.M., 2012. Study on coalbed methane accumulation 
characteristics and favorable areas in the Binchang area, southwestern Ordos Basin, China. International Journal of Coal Geology 95 (1), 1-11.

ХОДОТ, В.В. (Author), Song, S.Z., Wang, Y.А. (Translators), 1966. Coal and Gas Outburst. China Industry Press, Beijing, pp. 27-30. (in Chinese)

Yang, R.T., Saunders, J.T., 1985. Adsorption of gases on coals and heat treated coals at elevated temperature and pressure: 1. Adsorption from hydrogen and methane as single gases. Fuel 64 (5), 616-620.

Yao, Y.B., Liu, D.M., Tang, D.Z., Tang, S.H., Che, Y., Huang, W.H., 2009. Preliminary evaluation of the coalbed methane production potential and its geological controls in the Weibei Coalfield, Southeastern Ordos Basin, China. International Journal of Coal Geology 78 (1), 1-15.

Yee, D., Seidle, J.P., Hanson, W.B., 1993. Gas sorption on coal and measurement of gas content in hydrocarbons from coal. AAPG Studies in Geology 38, 203-218.

Zdravkov, A., Bechtel, A., Sachsenhofer, R.F., Kortenski, J., Gratzer, R., 2011. Vegetation differences and diagenetic changes between two Bulgarian lignite deposits - Insights from coal petrology and biomarker composition. Organic Geochemistry 42 (3), $237-254$.

Zhou, Y.P., Zhou, L., 2009. Fundamentals of high pressure adsorption. Langmuir 25 (3), 13461-13466. 